Theoretical study of phosphorescence in dye doped light emitting diodes

Boris Minaev, Emil Jansson, and Hans ÅgrenSigurd Schrader

Citation: The Journal of Chemical Physics 125, 234704 (2006); doi: 10.1063/1.2388263

View online: $\mathrm{http}: / / d x . d o i . o r g / 10.1063 / 1.2388263$

View Table of Contents: http://aip.scitation.org/toc/jcp/125/23

Published by the American Institute of Physics

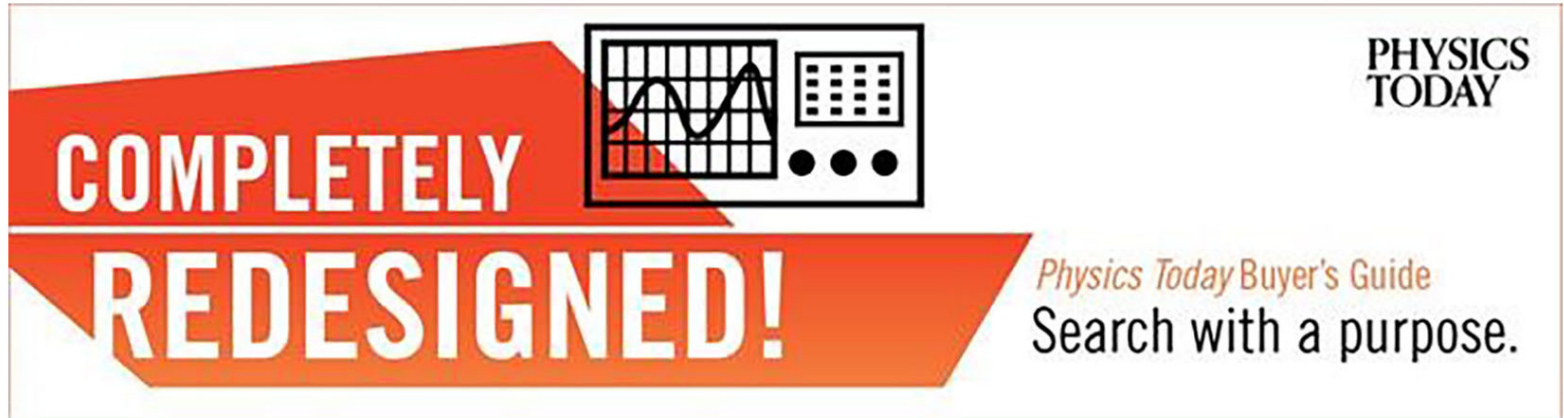




\title{
Theoretical study of phosphorescence in dye doped light emitting diodes
}

\author{
Boris Minaev, Emil Jansson, and Hans Ågren ${ }^{\text {a) }}$ \\ Laboratory of Theoretical Chemistry, The Royal Institute of Technology, SE-10691 Stockholm, Sweden \\ Sigurd Schrader \\ Technische Fachhochschule Wildau, University of Applied Sciences, D-15745 Wildau, Germany
}

(Received 29 November 2005; accepted 12 October 2006; published online 19 December 2006)

\begin{abstract}
Phosphorescence of platinum(II) octaethyl porphyrin (PtOEP), which has been used in organic light emitting diodes to overcome the efficiency limit imposed by the formation of triplet excitons, is studied by time-dependent (TD) density functional theory (DFT). The spin-orbit coupling (SOC) effects and the phosphorescence radiative lifetime $\left(\tau_{p}^{r}\right)$, calculated by the TD DFT method with the quadratic response technique, are analyzed for a series of porphyrins in order to elucidate the internal heavy atom effect on $\tau_{p}^{r}$. While the significance of the $d_{\pi}$ orbital admixture into the lowest unoccupied molecular orbital $e_{g}\left(\pi^{*}\right)$, proposed by Gouterman et al. [J. Chem. Phys. 56, 4073 (1972)], is supported by our SOC calculations, we find that the charge-transfer (CT) mechanism is more important; the CT state of the ${ }^{3} A_{2 g}$ symmetry provides effective SOC mixing with the ground state, and a large ${ }^{3} \mathrm{~A}_{2 g^{-}}{ }^{3} E_{u}$ transition dipole moment gives the main contribution to the radiative phosphorescence rate constant. The IR and Raman spectra in the ground state and first excited triplet state $\left(T_{1}\right)$ are studied for proper assignment of vibronic patterns. An orbital angular momentum of the $T_{1}$ state is not quenched completely by the Jahn-Teller effect. A large zero-field splitting is predicted for PtP and PtOEP which results from a competition between the SOC and Jahn-Teller effects. A strong vibronic activity is found for the $e_{g}$ mode at $230 \mathrm{~cm}^{-1}$ in PtP phosphorescence which is shifted to $260 \mathrm{~cm}^{-1}$ in PtOEP. This out-of-plane vibration of the Pt atom produces considerable change of the SOC mixing. The role of charge-transfer state of $d_{\pi} \pi^{*}$ type is stressed for the explanation of the electroluminescent properties of the dye doped light emitting diode.
\end{abstract}

(C) 2006 American Institute of Physics. [DOI: 10.1063/1.2388263]

\section{INTRODUCTION}

Poly- $N$-vinylcarbazol (PVC) and polyparaphenylene vinylene (PPV) constitute an important class of organic polymers, which can be doped by a heavy atom containing dye in order to increase electroluminescence (EL) of the composite material. ${ }^{1-6}$ This EL has attracted a wide interest because of the huge potential for application in display devices. ${ }^{3,6} \mathrm{~A}$ polymer light emitting diode (LED) device consists of a layer of a luminescent organic polymer, sandwiched between two metal electrodes. Electrons and holes are first injected from the electrodes into the polymer layer. These charge carriers then migrate through the organic layer, usually via interchain hopping processes, and eventually form intrachain excitons. ${ }^{3}$ Exciton formation in a polymer LED follows the Coulomb capture of nongeminate pairs of oppositely charged polarons. The process is similar to ion-radical recombination in solvents, where diffusion operates instead of interchain hopping. ${ }^{7-11}$ Consequently both the singlet and triplet excited states are formed during electron-hole recombination (both charge carriers have spin $S=\frac{1}{2}$ (singlet and triplet have $S$ equal to 0 and 1 , respectively). The triplet state has three spin projections $\left(M_{S}=0, \pm 1\right)$ and the singlet state has only one $\left(M_{S}=0\right)$ microstate. In the electron-hole encounter the spins are noncorrelated; ${ }^{7}$ thus spin statistics predicts that the

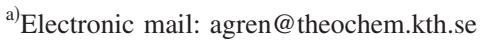

singlet-to-triplet ratio is 1:3. In organic semiconductors such as PVC and PPV only the singlet state can emit light, since the triplet state emission is spin forbidden. The ground state of the polymer is singlet; all chemical valencies are saturated, and the spins are paired in all chemical bonds. The singlet-singlet $(S-S)$ transition is dipole allowed and often provides intense luminescence. The triplet-singlet $(T-S)$ transition in the organic polymer is usually a million times less intense, since it gains the dipole activity through weak spinorbit coupling (SOC) perturbation. In organic $\pi$-conjugated molecules without heavy atoms the SOC matrix elements between the $S$ - $T$ states is about a thousand times smaller than the $S$ - $T$ energy gaps. ${ }^{12}$ In terms of perturbation theory the $T-S$ emission (phosphorescence) in such organic molecules is characterized by an extremely small electric dipole transition moment and by a very long radiative lifetime (about 1-100 s). Thus such phosphorescence cannot be seen at room temperature because the nonradiative quenching effectively competes with the light emission.

On this background there has been a generally accepted concept that the electroluminescence efficiency in organic LEDs is limited to $25 \%$ of the possible number of injected charge-carrier pairs. ${ }^{6}$ This concept, which assumes similar cross sections for the formation of $S$ and $T$ excitons, has recently come into question. ${ }^{1,3}$ The ratio has been investigated during the last five years and recent studies revealed that the singlet formation rate can be as high as $50 \%$ or even 
higher. $^{3,4,13}$ The high ratio is attributed to the larger cross section of singlet exciton formation than the triplet one due to delocalization in the charged particles in $\pi$-conjugated polymers. ${ }^{3,5}$ Even though the singlet exciton yield in $\pi$-conjugated polymers might be higher than the spinstatistics prediction, utilization of $T$ excitons in polymer LEDs can in principle significantly increase the light emission efficiency. ${ }^{1,3-6}$ Radiative recombination of both $S$ and $T$ states is the most attractive way to improve the performance of LEDs. In order to harvest light from triplet excitons the use of phosphorescent dyes has been proposed. ${ }^{1,3-6}$ These molecules incorporate a heavy metal atom to enforce the SOC between $S$ and $T$ states and to enhance phosphorescent $T \rightarrow S$ emission. Organic complexes with heavy transition metal atoms such as octaethyl-porphyrin platinum(II) (PtOEP) and tris(2-phenylpyridine) iridium $\left[\operatorname{Ir}(\mathrm{ppy})_{3}\right]$ were incorporated in the polymer as a dopant. ${ }^{1,5,6}$ The phosphorescent $T \rightarrow S$ emission of such polymers and that of other similar materials has been well characterized previously by lifetime measurements and by photoinduced $T-T$ absorption detection. ${ }^{4,12}$ The intramolecular phosphorescence radiative rate constant $\left(k_{p}\right)$ is higher than the fluorescence rate constant $\left(k_{f}\right)$; the efficiency of intersystem crossing (ISC) from singlet to triplet excited states is also enhanced. ${ }^{12,14-16}$ For example, the phosphorescence quantum yield $\left(\Phi_{p}\right)$ of $\operatorname{Pd}(\mathrm{II})$, $\mathrm{Rh}(\mathrm{III})$, and $\mathrm{Pt}(\mathrm{II})$ porphyrins is two to four orders of magnitude higher than the fluorescence quantum yield $\left(\Phi_{f}\right)$ in degassed solvents. ${ }^{17}$ All these porphyrins show very strong phosphorescence with lifetimes in the range of $1-0.1 \mathrm{~ms}^{17,18}$ For PdOEP the weak fluorescence is observed, while for PtOEP only a delayed fluorescence, produced by $T-T$ annihilation, has been reported. ${ }^{12,17}$ Similar photoluminescence (PL) and electroluminescence (EL) have been obtained for LED with platinum-containing polymers. ${ }^{1,2,4-6,19}$ An efficient polymer-based LED needs the development of a suitable polymer material that can achieve an effective energy transfer via Förster and Dexter mechanisms between the polymer host and the phosphorescent guest. $^{6}$ Baldo and Forrest ${ }^{2,19}$ have studied the $T-T$ energy transfer and $T-T$ annihilation in phosphorescent organic LEDs by comparison of transient EL with and without reverse bias after a short electrical pulse. They have found evidence of $T-T$ energy transfer for some combinations of guests and hosts, varying PtOEP and $\operatorname{Ir}(\mathrm{ppy})_{3}$ with different PVC-type polymers. ${ }^{2}$ Baldo and Forrest have also shown that the observed decrease in EL in such LEDs at high current density is due to $T-T$ annihilation. ${ }^{19}$ From the theory of $T$ $-T$ annihilation $^{20}$ it follows that the rate of the process strongly depends on the charge-transfer (CT) state properties, which in turn determine the rate of the electron-hole recombination. The low energy of the CT state provides an efficient interchain hopping of the charge carriers; at the same time it correlates with the high rate of the $T-T$ annihilation. This general idea, serving as an explanation of Baldo and Forrest results, ${ }^{2,19}$ can be illustrated by the following scheme. Let us assume that only the guest triplet state of the dyes $\left({ }^{3} M\right)$ participates in the $T-T$ annihilation,

$$
{ }^{3} M+{ }^{3} M \rightarrow{ }^{1}\left[{ }^{3} M \ldots{ }^{3} M\right] \rightarrow{ }^{1} M^{*}+{ }^{1} M,
$$

where ${ }^{1} M$ and ${ }^{1} M^{*}$ represent the ground and excited singlet states of the dye, respectively. The encounter of two triplet excitons produces delayed fluorescence ${ }^{1} M^{*} \rightarrow{ }^{1} M$ inside one dye moiety; its lifetime is twice as large in comparison with the triplet state lifetime. ${ }^{14}$ The collision complex of singlet multiplicity $\phi_{i}={ }^{1}\left[{ }^{3} M \cdots{ }^{3} M\right]$ can relax to the low-lying singlet state $\phi_{f}={ }^{1} M^{*}+{ }^{1} M$; the rate constant of this relaxation depends on the configuration interaction (CI) between the initial $\left(\phi_{i}\right)$ and final $\left(\phi_{f}\right)$ states. Calculations show that this $\mathrm{CI}$ is determined by exchange interaction and is usually very weak. $^{20}$ Nondirect mixing of the $\phi_{i}$ and $\phi_{f}$ states is induced through CI with the intermediate CT state ${ }^{1}\left[{ }^{2} M^{+} \ldots{ }^{2} M^{-}\right]$. For the homogeneous $T-T$ annihilation the resonance CT state ${ }^{1}\left[{ }^{2} M^{-} \cdot{ }^{2} M^{+}\right]$is also encountered. The CI mixing of the singlet states in Eq. (1) with the CT states includes large singleelectron integrals and finally provides a large matrix element between the $\phi_{i}$ and $\phi_{f}$ states. ${ }^{20}$ Injection of charges into the polymer chain leads to recombination of electrons ${ }^{2} P^{-}$and holes ${ }^{2} P^{+}$which creates the singlet and triplet excitons ${ }^{1,3}\left[{ }^{2} P^{+} \ldots{ }^{2} P^{-}\right] \rightarrow{ }^{1,3}\left[{ }^{1,3} P^{*} \cdots P\right]$. Both excitons and electronhole pairs can interact with the guest phosphorescent dye and can be trapped by the dye with subsequent phosphorescence emission. The former process is usually treated as the energy transfer of the Forster and Dexter mechanisms. ${ }^{1}$ The strength of the host-guest interaction should depend on the involvement of the intramolecular CT state inside the dye. CI calculations of the $T-T$ annihilation process also indicate an importance of the intramolecular and intermolecular CT states mixing. ${ }^{20}$ The physics behind these energy transfer processes is clear: separated charges create a strong Coulomb attraction between open-shell molecules and excitons (ion-radical pairs). In this paper we will show that the high phosphorescence efficiency of the PtOEP dye also depends on the lowlying CT state of $d_{\pi} \pi^{*}$ nature (charge transfer from the Pt ion to the porphin ring) which induces a strong SOC into the $T_{1}-S_{0}$ transition. Thus the electroluminescent properties of the PtOEP doped light emitting diodes are mutually connected: strong host-guest energy transfer, large phosphorescence rate constant, and efficient $T-T$ annihilation ${ }^{1,2}$ depend on the same peculiarity of the PtOEP electronic structure.

Porphyrin derivatives which contain zinc, palladium, and platinum ions are known to exhibit phosphorescence even at room temperature (zinc in a lesser extent). ${ }^{17,21}$ At the same time the closed shell porphyrins without heavy atoms show extremely weak phosphorescence though they have a very efficient nonradiative $S_{1} \rightsquigarrow T_{1}$ intersystem crossing. ${ }^{17,21,22}$ Photophysical properties of platinum porphyrins are not completely understood in spite of their fundamental significance for applications in light emitting diodes. ${ }^{2,18}$ The salient difference in luminescence of PtP and that of free-base porphin (FBP) including its complexes with light metals $(\mathrm{Mg}$, Al) cannot be easily explained by simple correlation with atomic numbers.

For FBP and light-metal porphyrins the $T_{1} \rightarrow S_{0}$ decay is almost completely dark in solid organic solvents, ${ }^{17}$ though these molecules have nitrogen atoms with lone pairs of electrons. Usually, such molecules exhibit strong phosphores- 
cence because of effective SOC-induced mixing between ${ }^{1} n \pi^{*}$ and ${ }^{3} \pi \pi^{*}$ states. ${ }^{14}$ The involvement of $n$ and $d$ electrons in porphyrin luminescence has been partly considered in recent studies ${ }^{16,23,24}$ by time-dependent density functional theory (TD DFT) calculations with quadratic response functions, ${ }^{25,26}$ including consideration of vibrational movements. $^{27,28}$

In this paper we are going to calculate the $T_{1} \rightarrow S_{0}$ transition dipole moment of platinum porphyrins by the TD DFT using the quadratic response $(\mathrm{QR})$ methodology in order to interpret the high efficiency of PtOEP-containing LED. We shall present also vibronic patterns of the $T_{1} \rightarrow S_{0}$ transitions together with a vibrational analysis of the ground and excited states. We wish to study the connections between electronic factors which are behind the large radiative probability of platinum porphyrins and their exciton hopping in LED polymers.

\section{METHOD OF CALCULATIONS}

Calculations of phosphorescence, excitation energies, and oscillator strengths for platinum- and palladiumporphyrins are carried out by TD DFT implemented in a recent version of the DALTON program. ${ }^{26}$ The advantages of the response theory have been earlier documented in applications to triplet state properties which depend on SOC. ${ }^{25}$ Linear and quadratic response functions in TD DFT are based on the ground state densities which are determined by solving the Kohn-Sham equations. ${ }^{29,30}$

$$
\begin{aligned}
& \delta E=\langle 0|[\delta \hat{\kappa}, \hat{H}]| 0\rangle=0 \\
& \hat{H}=\sum_{\sigma} \int_{\sigma} d \tau \hat{\Psi}_{\sigma}(\mathbf{r})^{\dagger} \frac{\delta E}{\delta \rho_{\sigma}(\mathbf{r})} \hat{\Psi}_{\sigma}(\mathbf{r}), \\
& \hat{\Psi}_{\sigma}(\mathbf{r})=\sum_{k} \phi_{k}(\mathbf{r}) a_{k \sigma} .
\end{aligned}
$$

Here $E\left[\rho_{\alpha}, \rho_{\beta}\right]$ is the chosen model functional with usual notations for the Kohn-Sham determinant

$$
|\tilde{0}\rangle=e^{-\hat{\kappa}}|0\rangle, \quad \hat{\kappa}_{r s}=\sum_{r s \sigma} \kappa_{r s} a_{r \sigma}^{\dagger} a_{s \sigma} .
$$

The Kohn-Sham orbitals $\phi_{k}$ form a representation of the ground state density

$$
\rho_{\sigma}=\left\langle 0\left|\hat{\Psi}(\mathbf{r})_{\sigma}^{\dagger} \hat{\Psi}_{\sigma}(\mathbf{r})\right| 0\right\rangle \equiv\langle 0|\hat{\rho}(\mathbf{r})| 0\rangle .
$$

The linear response method in DFT is described in Ref. 31. The influence of the SOC perturbation on the singlet-triplet $(S-T)$ transitions can be formulated in terms of QR functions. The SOC perturbation destroys the spin symmetry, and a nonvanishing $S-T$ transition dipole moment can be obtained as a residue of the QR function involving the combined perturbations of the spin-orbit coupling and the electric dipolephoton interactions.. ${ }^{25,32}$

Let us denote a perturbation operator by $\hat{V}$. The operator $\hat{H}+\hat{V}$ is now the Hamiltonian of the noninteracting system, which governs the time evolution of the Kohn-Sham orbitals. The ground state density can be expanded in a power series in the perturbation strength,

$$
\rho_{\sigma}=\rho_{\sigma}^{(0)}+\rho_{\sigma}^{(1)}+\rho_{\sigma}^{(2)} \ldots .
$$

One can assume that the zero-order ground state $\alpha$ and $\beta$ densities are equal.

Interpreting Eq. (5) as a parametrized time-evolution operator acting on the converged Kohn-Sham determinant, one can apply a time-dependent variational principle, which is based on the Ehrenfest theorem for an operator $Q$, to solve response equations for the $n$ th-order parameters $\hat{\kappa}^{(n)}$

$$
\delta^{n}\left\langle 0\left|\left[Q, e^{\hat{\kappa}}\left(\hat{H}\left[\rho_{\sigma}\right]+V-i \hbar \frac{\partial}{\partial t}\right) e^{-\hat{\kappa}}\right]\right| 0\right\rangle=0 .
$$

The $n$ th-order response functions, i.e., the $n$ th-order changes of a property, may then be identified from the expansion of property $A$

$$
\begin{aligned}
A= & \langle\hat{A}\rangle+\left\langle 0\left|\left[\hat{\kappa}^{(1)}, \hat{A}\right]\right| 0\right\rangle+\left\langle 0\left|\left[\hat{\kappa}^{(2)}, \hat{A}\right]\right| 0\right\rangle+\frac{1}{2}\langle 0| \\
& \times\left[\hat{\kappa}^{(1)},\left[\hat{\kappa}^{(1)}, \hat{A}\right]\right]|0\rangle \ldots .
\end{aligned}
$$

The quadratic response function in the frequency domain is given by

$$
\begin{aligned}
\langle\langle A ; V, V\rangle\rangle_{\omega_{1}, \omega_{2}}= & \left\langle 0\left|\left[\hat{\kappa}^{\omega_{1}, \omega_{2}}, \hat{A}\right]\right| 0\right\rangle \\
& +P_{12}\left\langle 0\left|\left[\hat{\kappa}^{\omega_{1}},\left[\hat{\kappa}^{\omega_{2}}, \hat{A}\right]\right]\right| 0\right\rangle,
\end{aligned}
$$

and the singlet-triplet transition amplitude is evaluated as the residue at a triplet state excitation energy with the dipole operator as the primary property and the spin-orbit operator as the perturber ${ }^{32}$ ( $X$ is an arbitrary triplet operator)

$$
\langle S|\mathbf{r}| T\rangle=\lim _{\omega_{2} \rightarrow \omega_{T}}\left(\omega_{2}-\omega_{T}\right) \frac{\left\langle\left\langle\mathbf{r} ; H_{\mathrm{SO}}, X\right\rangle\right\rangle_{0, \omega_{2}}}{\langle S|X| T\rangle} .
$$

The SOC matrix elements $\left\langle S_{0}\left|H_{\mathrm{SO}}\right| T_{1}\right\rangle$ can be evaluated as residues of linear response functions, and SOC between excited states are effectively obtained as residues of quadratic response functions. ${ }^{25,26}$

Phosphorescence of platinum porphyrins in low temperature solids ${ }^{33,34}$ results from vibronic transitions where an excitation of vibrational modes occurs simultaneously with the electronic $T_{1}-S_{0}$ transition. This vibronic fine structure contains information not only about the frequencies of the vibrational modes but also about SOC perturbations of three spin sublevels of the $T_{1}^{M_{S}}$ state and about their dependence on vibrational movement. To reveal this dependence is an important step in understanding phosphorescence. We shall concentrate our attention on PtP in comparison with other porphyrins.

For analysis of phosphorescence vibronic patterns we need to consider vibrational frequencies and modes of the ground state. The general expansion of the total wave function of the ground state in the adiabatic approximation $\Psi_{S_{0}, n}(q, Q)=S_{0}(q, Q) \Phi_{n}(Q)$ can be used, where $S_{0}$ is the electronic ground state wave function, and $q$ and $Q$ denote electronic and nuclear coordinates. ${ }^{35}$ In the harmonic approximation the nuclear wave function $\Phi_{n}(Q)$ is a product of 
wave functions of all vibrational modes $Q_{i}$. Only one of them is considered to be excited in the state with $n=1$ quantum number, thus we neglect combinational lines and overtones. In such approach the $0-n$ vibronic line intensity is determined by the following $T_{1}^{a}-S_{0}$ transition dipole moment ${ }^{27}$

$$
\begin{aligned}
& \left\langle T_{1}^{a}(0)|\mathbf{q}+\mathbf{Q}| S_{0}(n)\right\rangle \\
& \quad=\left\langle T_{1}^{a}|\mathbf{q}| S_{0}\right\rangle_{0}\langle 0 \mid n\rangle+\frac{\partial M_{T, S}^{a}(Q)}{\partial Q_{i}}\left\langle\Phi_{0}\left|\mathbf{Q}_{i}\right| \Phi_{n}\right\rangle,
\end{aligned}
$$

where

$$
M_{T, \mathbf{S}}^{a}(Q)=\left\langle T_{1}^{a}(q, Q)|\mathbf{q}| S_{0}(q, Q)\right\rangle .
$$

The first term corresponds to the Franck-Condon mechanism. Quantization of the spin projection is implemented here for the zero-field splitting (ZFS) approach $^{14}$ and coincides with molecular symmetry axes; $T^{a}$ means a spin sublevel with the total spin projection on the $a$ axis equal to zero.

The geometry optimization, IR and Raman spectra, and general analysis of excited states are carried out with the Lanl2DZ basis with effective core potential. ${ }^{36}$ The TD DFT QR calculations of the $S$ - $T$ transition moments in all porphyrins and their vibrational mode dependence are performed with the splitted 3-21G basis set; ${ }^{37}$ for QR calculations of $\mathrm{PtP}$ we have used for platinum atom the so-called welltempered basis set (WTBS) of Huzinaga and Klobukowski. ${ }^{39}$ The Franck-Condon (FC) factors are calculated through the excited state gradients in the B3LLYP/Lanl2DZ approach with the code developed by Macak et al. $^{39}$ SOC integral calculations are performed with two approximations: scaled atomic charges proposed by Koseki et al. ${ }^{40}$ and with atomic mean field SOC two-electron integrals (AMFI). ${ }^{41}$ Massweighted displacements along the active normal modes of $e_{g}$ symmetry are used for vibronic calculations.

\section{RESULTS AND DISCUSSION}

First we shall start with an analysis of the molecular structure in the ground and first excited states and with their vibrational patterns. The PtOEP molecule has a number of conformers with different orientations of the ethyl groups. One such conformer optimized by the B3LYP/Lanl2DZ method is presented in Fig. 1. It is close to the triclinic B ruffled structure of NiOEP optimized by Stoll et al. $^{42}$ The symmetric PtOEP isomer with an all-trans orientation of ethyl groups ${ }^{43}$ is found with one imaginary frequency. A few other isomers (with clockwise, alternating and bialternating positions of the ethyl groups) $)^{43}$ are obtained with similar energies; they all have almost identical electronic absorption spectra calculated by TD DFT (all differences are within the $0.1 \mathrm{eV}$ margin), and they are similar to the spectrum of the PtP molecule besides a small redshift $(0.05-0.08 \mathrm{eV})$ of the formers. The different geometries of the peripheral ethyl substituents have no significant effect on the molecular orbitals (MOs), which practically coincide with the MOs shown in Figure 2 for PtP. Thus we will restrict our discussion to the PtP molecule instead of giving a tiresome analysis of the isomers of PtOEP; some important spectral peculiarities will be specially mentioned. A comparison of calculated elec-

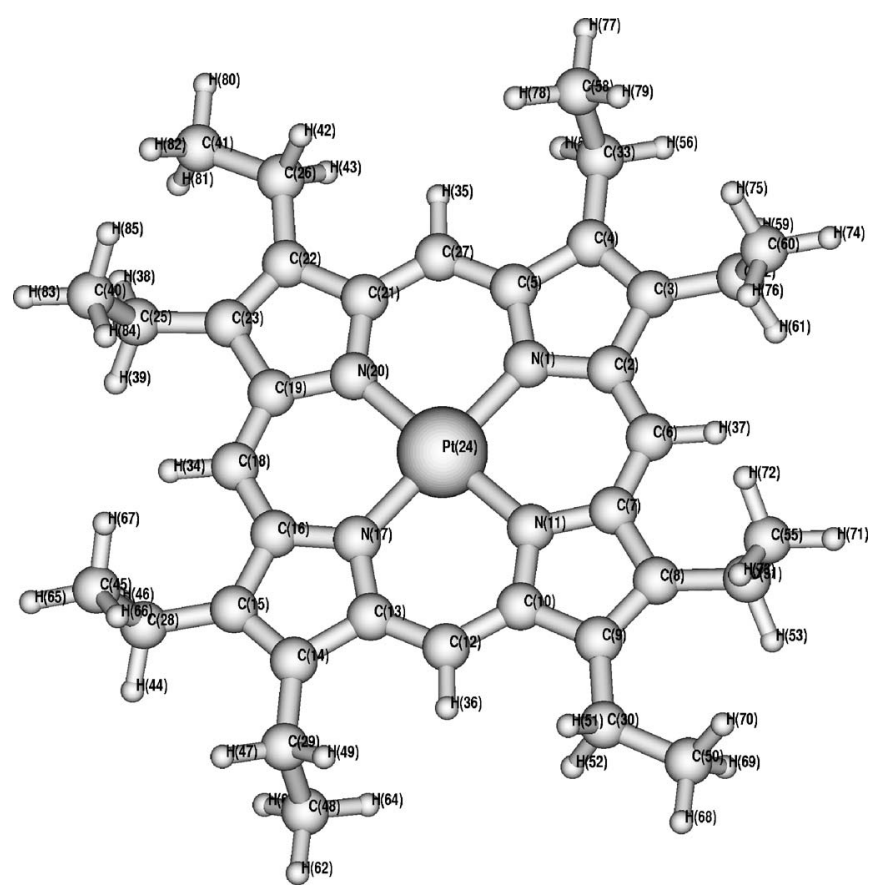

FIG. 1. Numeration of atoms in PtOEP molecule.

tronic spectra for the series of porphyrins is given in Tables I and II.

Experimental information on structure and vibrational spectra of PtP and PtOEP is rather scarce. ${ }^{18,44}$ It refers to different crystals and solvents at various temperatures ${ }^{17,18}$ and cannot be conclusive. Thus we have to compare the PtP structure with the well-studied Zn-porphyrin (ZnP) molecule. ${ }^{27,45,47}$ The optimized geometries of PtP and ZnP are presented in Table III together with the PdP structure, which is the closest relative to platinum porphyrin.

In the present work the ground state and the lowest triplet excited state optimized geometry and vibrational modes are used for the $T_{1} \rightarrow S_{0}$ transition moment and the phosphorescence radiative lifetime calculations. The geometry was optimized with the B3LYP functional and Lanl2dz basis set. ${ }^{36}$ The geometry is found to retain $D_{2 h}$ symmetry in the lowest triplet excited state and $D_{4 h}$ symmetry in the ground state with a positive definite Hessian.

The structures of FBP and ZnP molecules are quite similar to geometries found in other DFT studies with complete basis sets. $^{23,27,46,48}$ The triplet excited ZnP, PdP, and PtP molecules have similar trends in their frequency shift. In general the Lanl2DZ basis set provides slightly higher vibrational frequencies in comparison with the $6-31 \mathrm{G}^{* *}$ basis set (Tables IV and VII), but the difference does not exceed a few percent. Taking into account the scaling factor (0.967), the agreement with experimental IR and Raman spectra for NiP, $\mathrm{ZnP}$, and free-base porphin is very reasonable. ${ }^{27,48}$ All molecules ( $\mathrm{ZnP}, \mathrm{PdP}$, and PtP) are planar in both the $S_{0}$ and $T_{1}$ states. Pd- and Pt-porphyrins are predicted with the singlet ground state as it is known from experiment.

The effective core potential (ECP) basis set Lanl2DZ provides similar structures for $\mathrm{ZnP}$ like the more sophisticated full basis sets $\left(6-31 \mathrm{G}^{* *}, 6-311 \mathrm{G}^{* *}\right) \mathrm{do},{ }^{27}$ but slightly overestimates some distances (Table III). In $\mathrm{ZnP}$ the 

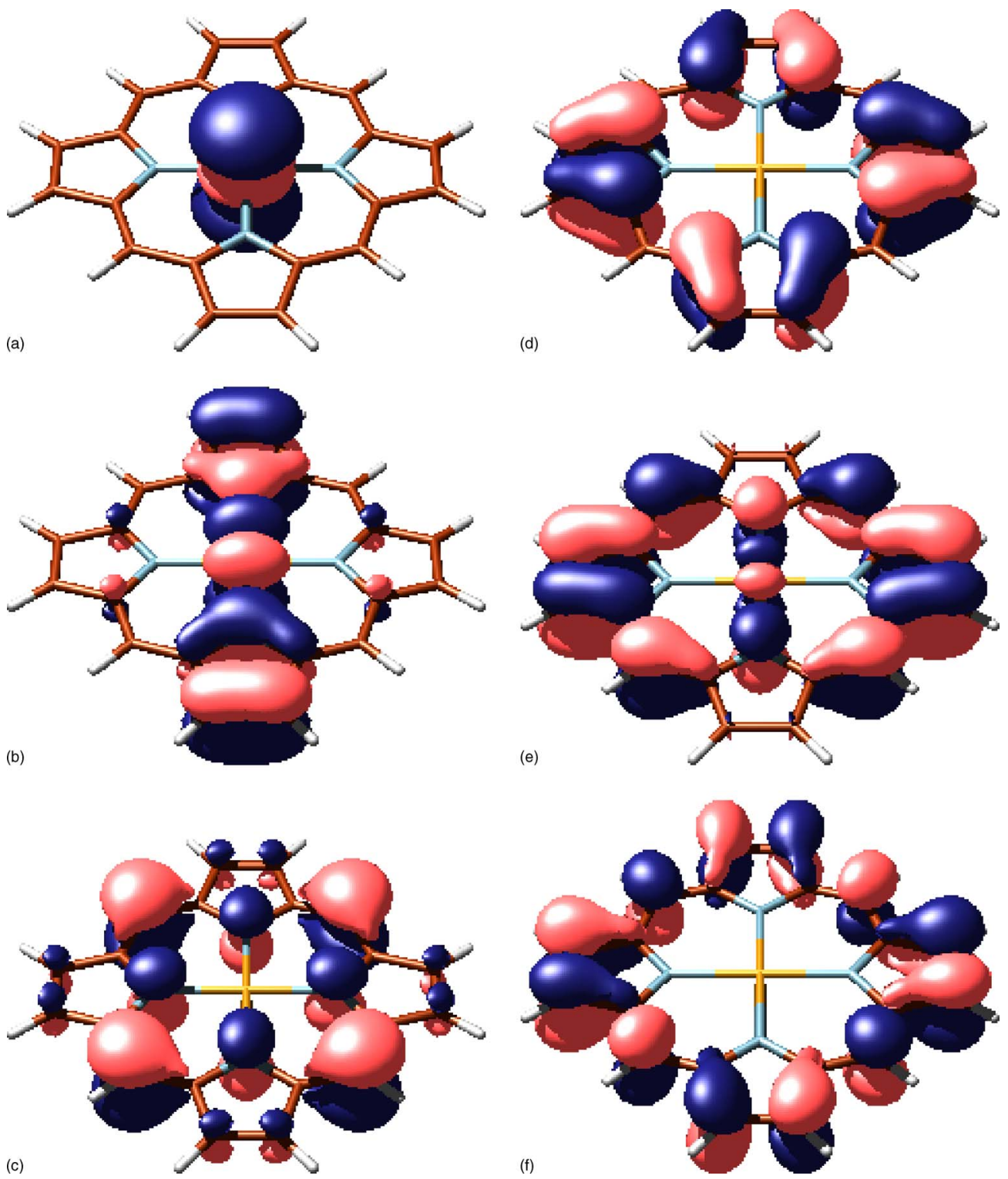

FIG. 2. Molecular orbitals of Pt-porphyrin: (a) $\psi_{85}$, (b) $\psi_{87}$, (c) $\psi_{88}$, (d) $\psi_{89}$, (e) $\psi_{90}$, and (f) $\psi_{92}$.

Lanl2DZ result for the $\mathrm{Zn}-\mathrm{N}$ distance $(2.068 \AA)$ is close to the distance optimized by Nguyen et al. ${ }^{49}$ with the $6-31 \mathrm{G}^{*}$ basis $(2.056 \AA)$, but still overestimates the experimental value (Table III). It is interesting to note that in $\mathrm{ZnP}$ the metal-nitrogen distance is much larger than in $\operatorname{PtP}(2.043 \AA)$ in spite of the fact that their ionic radii $\left(r_{i}\right)$ in crystals are close; for coordination number 4 both $\mathrm{Zn}^{2+}$ and $\mathrm{Pt}^{2+}$ ions have $r_{i}=0.6 \AA \AA^{50}$ This is in agreement with the contention of Jarzecki et $a l^{47}$ that the size of the porphyrin cavity is larger than formerly assumed ${ }^{51}$ and that even heavy metals can fit the cavity without ruffling or altering the stereochemistry of the porphyrin core.

\section{A. Calculation of IR and Raman spectra of the ground state and the lowest triplet states}

Vibrational IR and Raman spectra of porphyrins in their ground state have been studied by numerous investigators (for a review see Ref. 44). Gladkov and Solovyov ${ }^{52,53}$ and $\mathrm{Li}$ and Zgierski $^{44}$ developed a most accurate empirical force field for metal-porphyrins, which reproduces quite well the IR and Raman resonance spectra of a number of isotopomers including NiP, CuP, and ZnP. Pulay and co-workers ${ }^{47,54,55}$ have measured nonresonant Raman spectra of $\mathrm{ZnP}$ and $\mathrm{MgP}$ molecules and calculated the scaled quantum mechanical (SQM) force fields. They have assigned many vibrational frequencies with the B3LYP/6-31G* approach. In Tables IV and VI we present our results of vibrational analysis obtained by B3LYP/Lanl2DZ calculations for the ground state of the $\mathrm{ZnP}$ and PtP molecules without scaling factors. $\mathrm{ZnP}$ is chosen for comparison since its vibrational data set is relatively more complete than the others. ${ }^{44}$

IR spectra of metastable triplet states have been the subject of numerous experimental investigations. ${ }^{56,57}$ Since a large concentration of the triplet excited PtOEP molecules can be achieved in LED polymers, it seems to be possible to detect the IR spectrum in such triplet states by time-resolved infrared spectroscopy. For the future test of such an experiment we present in Table $\mathrm{V}$ the calculated IR spectrum of 
TABLE I. Electronic absorption spectrum of the ZnP, PdP, and PtP molecules calculated by the B3LYP/ Lanl2DZ method. $\Delta E$ is excitation energy $(\mathrm{eV})$, oscillator strengths $(f)$ are given in parentheses.

\begin{tabular}{|c|c|c|c|c|c|c|}
\hline \multirow[b]{2}{*}{ State } & \multicolumn{2}{|c|}{$\mathrm{ZnP}$} & \multicolumn{2}{|c|}{$\mathrm{PdP}$} & \multicolumn{2}{|c|}{ PtP } \\
\hline & Calc. & Expt. $^{a}$ & Calc. & Expt. $^{b}$ & Calc. & Expt. ${ }^{\mathrm{b}, \mathrm{c}}$ \\
\hline $1{ }^{3} E_{u}$ & 1.68 & 1.82 & 1.83 & 1.95 & 1.94 & 2.02 \\
\hline $2{ }^{3} E_{u}$ & 2.07 & $\cdots$ & 2.19 & $\cdots$ & 2.25 & $\cdots$ \\
\hline $1{ }^{1} E_{u}$ & $2.39(0.0001)$ & $2.18(0.005)$ & $2.52(0.001)$ & $2.34(0.08)$ & $2.60(0.0033)$ & $2.39(0.09)$ \\
\hline $2{ }^{1} E_{u}$ & $3.50(2 \times 0.89)$ & $3.22(1.39)$ & $3.47(2 \times 0.74)$ & $3.19(1.6)$ & $3.53(2 \times 0.72)$ & $3.33(1.8)$ \\
\hline $3{ }^{1} E_{u}$ & $3.74(2 \times 0.081)$ & $3.81(0.15)$ & $3.93(2 \times 0.037)$ & & $4.05(2 \times 0.07)$ & $4.01(0.1)$ \\
\hline $1{ }^{1} A_{2 g}$ & $3.66(0)$ & $\ldots$ & $3.00(0)$ & $\ldots$ & $2.67(0)$ & $\ldots$ \\
\hline $1{ }^{1} B_{2 g}$ & $3.70(0)$ & $\cdots$ & $3.06(0)$ & $\ldots$ & $2.74(0)$ & $\ldots$ \\
\hline $1{ }^{1} E_{g}$ & $3.77(0)$ & $\cdots$ & $3.27(0)$ & $\cdots$ & $3.11(0)$ & $\cdots$ \\
\hline
\end{tabular}

${ }^{\mathrm{a}}$ Reference 78 .

${ }^{\mathrm{b}}$ Reference 33. In EPAF solvent (diethylether, isopentane, dimethylformamode, and ethanol in the 12:10:6:1 ratio).

${ }^{\mathrm{c}}$ Reference 18 For PtOEP in solid film.

PtP in the first triplet ${ }^{3} B_{3 u}$ excited state. Because of the JahnTeller effect in the triplet state of PtP its symmetry is reduced from $D_{4 h}$ to $D_{2 h}$ and the $e_{u}$ modes are split into $b_{2 u}$ and $b_{3 u}$ modes. A correlation of irreducible representations of the two point groups is given in Table VI.

In Fig. 3 and 4 we present the simulated IR and Raman spectra of the PtP molecule in the ground singlet and in the first excited triplet states. The scaling factor (0.967) is used here in order to make a comparison with possible experimental data. In Table VII only "gerade" modes are presented as these are active in the Raman spectra and in vibronic emission spectra for PtP molecule. Li and Zgierski ${ }^{44}$ did not consider the out-of-plane vibrations when they introduced their numeration of vibrational modes. Thus we need to use our own numeration thoughout in Tables VII and IV. A large increase of the Raman scattering intensity (two to three or- ders of magnitude) is predicted for many $b_{1 g}$ vibrational modes ( $D_{2 h}$ symmetry) of the PtP molecule in the $T_{1}$ state. This is probably connected with a small ${ }^{3} B_{3 u}{ }^{3} B_{2 u}$ energy gap at the $T_{1}$ state optimized geometry. The outstanding feature in this respect is $\nu_{93}$ vibration in Table VII. Its Raman intensity rises to $1640 \AA^{4} / \mathrm{amu}$ (the frequency decreases only by $\left.4 \mathrm{~cm}^{-1}\right)$. This in-plane vibration includes $\mathrm{C}_{\alpha}-\mathrm{N}-\mathrm{C}_{\alpha}$ asymmetric stretches in the pyrrole rings with notable $\mathrm{N}-\mathrm{Pt}-\mathrm{N}$ distortions. Comparison of the calculated IR spectra of PtP in the $S_{0}$ and $T_{1}$ states indicates some intensity redistributions (Fig. 3). For the out-of-plane vibrations $\left(b_{1 u}\right)$ some new weak bands occur with very low intensity (former forbidden $b_{2 u}$ modes in the $D_{4 h}$ point group of the ground state).

The fundamental intense IR bands $\left(\nu_{38}\right.$ and $\nu_{49}$ in Table IV) are only slightly shifted and are enhanced in the triplet

TABLE II. Quadratic response DFT/B3LYP calculations of phosphorescence intensity at the ground state optimized geometry. $\Delta E$ is the transition energy (eV), electric dipole $S_{0}-T_{1}$ transition moments are in a.u. $\left(e a_{0}\right)$, $k_{b}^{a}\left(\mathrm{~s}^{-1}\right)$ is the rate constants for emission from the $T_{1}^{a}$ spin sublevel, polarized along the $b$ axis; $\tau_{p}(L)$ (s) is the phosphorescent radiative lifetime at the low-temperature limit $(4 \mathrm{~K}), \tau_{p}(H)$ is the phosphorescent radiative lifetime at high-temperature limit $(77 \mathrm{~K})$. The $z$ axis is perpendicular to the plane. QR calculations are done with the $3-21 \mathrm{G}$ basis set. For the Pt atom the WTBS basis set of Huzinaga and Klobukowski (Ref. 38) is used.

\begin{tabular}{|c|c|c|c|c|c|c|c|}
\hline Molecule & $\Delta E$ & $\left\langle{ }^{3} \Psi_{1}^{x}|z|^{1} \Psi_{0}\right\rangle$ & $\left\langle{ }^{3} \Psi_{1}^{z}|x|^{1} \Psi_{0}\right\rangle$ & $k_{z}^{x}$ & $k_{x}^{z}$ & $\tau_{p}(L)$ & $\tau_{p}(H)$ \\
\hline $\operatorname{FBP}\left({ }^{3} B_{2 u}\right)^{\mathrm{a}}$ & 1.46 & $2.4 \times 10^{-5}$ & $6.0 \times 10^{-7}$ & 0.0018 & $0.6 \times 10^{-6}$ & 540 & 1619 \\
\hline FBP,Expt. ${ }^{b}$ & & & & 0.002 & & 500 & \\
\hline FBP,Expt. ${ }^{c, d}$ & 1.58 & & & 0.0078 & & & $>70$ \\
\hline $\operatorname{MgP}\left({ }^{3} E_{u}\right)^{\mathrm{a}}$ & 1.72 & $6.6 \times 10^{-5}$ & $1.2 \times 10^{-6}$ & 0.024 & $1.0 \times 10^{-5}$ & 42.4 & 127 \\
\hline MgP,Expt. ${ }^{\mathrm{d}}$ & 1.70 & & & 0.021 & & & 47 \\
\hline $\mathrm{ZnP}\left({ }^{3} E_{u}\right)^{\mathrm{a}}$ & 1.81 & $7.1 \times 10^{-5}$ & $1.80 \times 10^{-4}$ & 0.031 & 0.203 & 4.9 & 12.8 \\
\hline ZnP,Expt. $^{\mathrm{e}}$ & 1.82 & & & $0.16^{\mathrm{c}}$ & $1.0^{\mathrm{c}}$ & & $<10$ \\
\hline $\operatorname{PtP}\left({ }^{3} E_{u}\right)$ & 1.95 & $1.6 \times 10^{-2}$ & $3.0 \times 10^{-2}$ & $1.98 \times 10^{3}$ & $6.98 \times 10^{3}$ & $1.43 \times 10^{-4}$ & $3.34 \times 10^{-4}$ \\
\hline PtP,Expt. ${ }^{\mathrm{f}}$ & 2.02 & & & $0.2^{\mathrm{c}}$ & $1.0^{\mathrm{c}}$ & & $\approx 1 \times 10^{-4}$ \\
\hline
\end{tabular}

${ }^{\mathrm{a}}$ Reference 27.

${ }^{\mathrm{b}}$ Reference 77.

${ }^{\mathrm{c}}$ Reference 64; relative $k^{a}$ values are presented.

${ }^{\mathrm{d}}$ Reference 22 , averaged $k$ value is measured at $77 \mathrm{~K}$.

${ }^{\mathrm{e}}$ References 66 and 67.

${ }^{\mathrm{f}}$ References 34, 64, and 72 . 
TABLE III. Bond lengths ( $\AA$ ) calculated by the DFT B3LYP/Lanl2DZ method for the ground state and for the lowest triplet excited state of the PtP, PdP, and $\mathrm{ZnP}$ molecules.

\begin{tabular}{|c|c|c|c|c|c|c|c|}
\hline \multirow[b]{2}{*}{ Bond } & \multicolumn{2}{|c|}{ Pt-P } & \multicolumn{2}{|c|}{ Pd-P } & \multicolumn{3}{|c|}{$\mathrm{Zn}-\mathrm{P}$} \\
\hline & ${ }^{1} A_{1 g}^{\mathrm{a}}$ & ${ }^{3} B_{1 u}{ }^{a}$ & ${ }^{1} A_{1 g}{ }^{\mathrm{a}}$ & ${ }^{3} B_{1 u}{ }^{\mathrm{a}}$ & ${ }^{1} A_{1 g}{ }^{a}$ & ${ }^{3} B_{1 u}^{\mathrm{a}}$ & ${ }^{1} A_{1 g}:$ Expt. ${ }^{\mathrm{b}}$ \\
\hline$\beta^{\prime}-\beta^{\prime} \mathrm{C}_{5}-\mathrm{C}_{7}$ & 1.375 & 1.373 & 1.375 & 1.373 & 1.379 & 1.376 & 1.354 \\
\hline$\beta-\beta \mathrm{C}_{17}-\mathrm{C}_{18}$ & 1.375 & 1.400 & 1.375 & 1.401 & 1.379 & 1.404 & 1.354 \\
\hline$\alpha^{\prime}-\beta^{\prime} \mathrm{C}_{5}-\mathrm{C}_{1}$ & 1.453 & 1.456 & 1.455 & 1.458 & 1.458 & 1.461 & 1.441 \\
\hline$\alpha-\beta \mathrm{C}_{13}-\mathrm{C}_{17}$ & 1.453 & 1.428 & 1.455 & 1.429 & 1.458 & 1.431 & 1.441 \\
\hline$\alpha^{\prime}-m \mathrm{C}_{1}-\mathrm{C}_{9}$ & 1.395 & 1.391 & 1.397 & 1.392 & 1.405 & 1.399 & 1.389 \\
\hline$\alpha-m \mathrm{C}_{13}-\mathrm{C}_{9}$ & 1.395 & 1.423 & 1.397 & 1.425 & 1.405 & 1.433 & 1.389 \\
\hline$\alpha-N \mathrm{C}_{14}-\mathrm{N}$ & 1.393 & 1.393 & 1.390 & 1.391 & 1.391 & 1.393 & 1.371 \\
\hline$\alpha^{\prime}-N \mathrm{C}_{1}-\mathrm{N}$ & 1.393 & 1.394 & 1.390 & 1.393 & 1.391 & 1.396 & 1.371 \\
\hline$\beta^{\prime}-\mathrm{H} \mathrm{C}_{5}-\mathrm{H}_{31}$ & 1.082 & 1.082 & 1.082 & 1.082 & 1.082 & 1.082 & \\
\hline$\beta-\mathrm{H} \mathrm{C}_{17}-\mathrm{H}_{24}$ & 1.082 & 1.082 & 1.082 & 1.082 & 1.082 & 1.082 & \\
\hline$m-\mathrm{H} \mathrm{C}_{9}-\mathrm{H}_{27}$ & 1.086 & 1.087 & 1.086 & 1.087 & 1.087 & 1.088 & \\
\hline $\mathrm{M}_{37}-\mathrm{N}_{34}$ & 2.043 & 2.062 & 2.049 & 2.067 & 2.068 & 2.085 & 2.04 \\
\hline $\mathrm{M}_{37}-\mathrm{N}_{36}$ & 2.043 & 2.057 & 2.049 & 2.064 & 2.068 & 2.078 & 2.04 \\
\hline
\end{tabular}

${ }^{\mathrm{a}}$ This work.

${ }^{\mathrm{b}}$ Reference 46.

state IR spectrum $\left(b_{1 u}\right.$ vibrations at 785.9 and $899.6 \mathrm{~cm}^{-1}$ in Table V). The main change upon $S-T$ excitation is connected with $\nu_{73,74}=1352 \mathrm{~cm}^{-1}$ degenerate $e_{u}$ mode (Table IV); in the excited triplet state it is split to the $b_{3 u}$ component $\left(1357 \mathrm{~cm}^{-1}\right.$ in Table V) and is enhanced 14 times. This mode corresponds to pyrrole symmetric half-ring stretching $\left(\nu_{41}\right.$ in

TABLE IV. The infrared active spectra of Pt- and Zn-porphyrin calculated by the B3LYP DFT method with the Lanl2DZ basis set. IR intensity is in $\mathrm{km} / \mathrm{mole}$; frequency is in $\mathrm{cm}^{-1}$.

\begin{tabular}{|c|c|c|c|c|c|c|c|c|}
\hline \multirow[b]{2}{*}{ Symmetry } & \multicolumn{4}{|c|}{ Zn-P } & \multicolumn{4}{|c|}{ Pt-P } \\
\hline & $\mathrm{i}^{\mathrm{a}}$ & $\nu_{i}$ & Intensity & $\nu_{i}$ Expt. $^{\mathrm{b}}$ & i & $\nu_{i}$ & Intensity & $\nu_{i}$ Expt. $^{\mathrm{C}}$ \\
\hline$e_{u}$ & 12,13 & 241.4 & 0.4 & & 7,8 & 242.3 & 1.0 & \\
\hline$e_{u}$ & 16,15 & 354.9 & 6.7 & & 16,17 & 389.7 & 0.1 & \\
\hline$e_{u}$ & 19,20 & 393.0 & 7.5 & & 19,20 & 411.9 & 6.1 & \\
\hline$e_{u}$ & 37,36 & 753.4 & 17.0 & 739 & 36,37 & 755.5 & 10.5 & 740 \\
\hline$e_{u}$ & 41,42 & 816.9 & 4.8 & 799 & 43,44 & 826.1 & 6.5 & 798 \\
\hline$e_{u}$ & 54,55 & 1009.0 & 71.4 & 993 & 56,57 & 1033.8 & 57.4 & 995 \\
\hline$e_{u}$ & 59,60 & 1028.9 & 0.1 & 1019 & 59,60 & 1063.6 & 1.1 & 1023 \\
\hline$e_{u}$ & 62,63 & 1093.4 & 84.0 & 1052 & 64,63 & 1102.6 & 101.7 & 1054 \\
\hline$e_{u}$ & 67,68 & 1183.9 & 9.0 & 1151 & 67,68 & 1193.1 & 5.5 & 1151 \\
\hline$e_{u}$ & 71,72 & 1276.0 & 0.1 & & 71,72 & 1301.8 & 1.4 & \\
\hline$e_{u}$ & 73,74 & 1330.7 & 10.8 & 1299 & 73,74 & 1352.1 & 29.6 & 1304 \\
\hline$e_{u}$ & 80,81 & 1420.6 & 5.4 & 1384 & 80,79 & 1429.1 & 0.0 & 1384 \\
\hline$e_{u}$ & 84,83 & 1467.3 & 4.8 & 1438 & 82,83 & 1493.1 & 15.4 & 1442 \\
\hline$e_{u}$ & 87,88 & 1556.7 & 20.0 & 1517 & 87,86 & 1583.6 & 9.2 & 1517 \\
\hline$e_{u}$ & 90,91 & 1596.4 & 4.2 & 1558 & 90,91 & 1635.0 & 10.5 & 1563 \\
\hline$e_{u}$ & 95,96 & 3205.6 & 11.7 & & 95,96 & 3215.0 & 8.7 & \\
\hline$e_{u}$ & 99,100 & 3253.2 & 3.3 & & 99,100 & 3258.0 & 1.9 & \\
\hline$e_{u}$ & 103,104 & 3279.2 & 32.2 & & 103,104 & 3283.8 & 24.2 & \\
\hline$a_{2 u}$ & 2 & 73.8 & 1.3 & & 2 & 64 & 0.1 & \\
\hline$a_{2 u}$ & 6 & 196.0 & 41.3 & & 6 & 148 & 29.3 & \\
\hline$a_{2 u}$ & 17 & 357.1 & 13.2 & & 15 & 343 & 7.0 & \\
\hline$a_{2 u}$ & 33 & 732.4 & 40.4 & 699 & 34 & 714 & 8.8 & 690 \\
\hline$a_{2 u}$ & 38 & 801.7 & 121.0 & 765 & 38 & 779 & 69 & 764 \\
\hline$a_{2 u}$ & 49 & 919.1 & 239.5 & 849 & 49 & 879 & 122 & 829 \\
\hline
\end{tabular}

${ }^{\mathrm{a}}$ Through numeration of all modes.

${ }^{\mathrm{b}}$ Reference 47.

${ }^{\mathrm{c}}$ Reference 79. 
TABLE V. Infrared spectrum calculated for the lowest triplet ${ }^{3} B_{1 u}$ state of platinum porphyrin by the UB3LYP/ Lanl2DZ method. "Int." means IR absorption intensity ( $\mathrm{km} / \mathrm{mole})$; frequency $(\nu)$ is in $\mathrm{cm}^{-1}$

\begin{tabular}{|c|c|c|c|c|c|}
\hline \multicolumn{2}{|c|}{$b_{1 u}$} & \multicolumn{2}{|c|}{$b_{2 u}$} & \multicolumn{2}{|c|}{$b_{3 u}$} \\
\hline$\nu$ & Int. & $\nu$ & Int. & $\nu$ & Int. \\
\hline 55.1 & 0.1 & 233.7 & 3.1 & 234.1 & 0.1 \\
\hline 81.2 & 4.5 & 373.2 & 2.5 & 378.6 & 0.6 \\
\hline 252.9 & 4.9 & 400.0 & 22.3 & 403.4 & 1.6 \\
\hline 297.7 & 2.8 & 741.8 & 20.3 & 754.6 & 13.3 \\
\hline 348.8 & 13.1 & 824.4 & 6.9 & 831.4 & 37.6 \\
\hline 684.2 & 3.3 & 1040.1 & 77.3 & 999.4 & 107.0 \\
\hline 723.5 & 73.9 & 1053.3 & 2.8 & 1058.6 & 2.4 \\
\hline 785.9 & 123.5 & 1066.2 & 77.4 & 1094.4 & 79.0 \\
\hline 807.3 & 6.9 & 1182.2 & 64.3 & 1191.8 & 27.7 \\
\hline \multirow[t]{9}{*}{899.6} & 183.8 & 1316.5 & 4.0 & 1290.8 & 41.3 \\
\hline & & 1333.9 & 0.0 & 1357.6 & 410.3 \\
\hline & & 1379.6 & 19.5 & 1396.1 & 29.7 \\
\hline & & 1415.9 & 1.6 & 1484.5 & 84.3 \\
\hline & & 1467.9 & 29.7 & 1535.9 & 0.1 \\
\hline & & 1611.0 & 19.1 & 1609.7 & 136.4 \\
\hline & & 3211.1 & 0.7 & 3211.8 & 24.7 \\
\hline & & 3258.9 & 0.2 & 3259.4 & 5.7 \\
\hline & & 3282.5 & 23.9 & 3284.7 & 22.7 \\
\hline
\end{tabular}

the classification of Ref. 44). It includes Pt-N(33) and Pt-N(34) out-of-phase stretching vibrations; that is why its frequency changes much in comparison with the $\mathrm{ZnP}$ molecule (Table IV).

Comparison with the previously calculated free-base porphin molecule in a large basis set ${ }^{27}$ indicates similarity in structural and IR spectral changes upon $S_{0} \rightarrow T_{1}$ excitation. In both molecules the largest structural deformations occur in one group of pyrrole rings: the $\mathrm{C}_{\beta}-\mathrm{C}_{\beta}$ bond length increases and the the $\mathrm{C}_{\alpha}-\mathrm{C}_{\beta}$ bond length decreases (by about $0.025 \AA$ ). A similar large bond length increase is obtained for one shoulder of the $\mathrm{C}_{\alpha}-\mathrm{C}_{m}$ methine bridge, while the other one is almost nonchanged (Table II).

The most prominent change in the IR spectra of freebase porphin is connected with the $\nu_{72}\left(b_{2 u}\right)$ vibration, the intensity of which increases from $57 \mathrm{~km} / \mathrm{mole}$ to $325 \mathrm{~km} /$ mole upon $S-T$ excitation. ${ }^{27}$ This provides a very intense peak in the IR absorption spectrum of the triplet state like in the PtP molecule (Fig. 3). There are also some differences: in the FBP molecule the frequency of this mode de-

TABLE VI. Correlation of vibrational symmetry between the ground state of platinum-porphyrin ( $D_{4 h}$ point group) and the triplet excited state $\left(D_{2 h}\right.$ point group) of the same molecules.

\begin{tabular}{ccccc}
\hline \hline \multicolumn{2}{c}{ In plane } & & \multicolumn{2}{c}{ Out of plane } \\
\cline { 1 - 2 } \cline { 4 - 5 }$S_{0}$ & $T_{1}$ & & $S_{0}$ & $T_{1}$ \\
\hline$e_{u}$ & $b_{2 u}$ & & $e_{g}$ & $b_{2 g}$ \\
$e_{u}$ & $b_{3 u}$ & $e_{g}$ & $b_{3 g}$ \\
$a_{1 g}$ & $a_{g}$ & $a_{1 u}$ & $a_{u}$ \\
$b_{1 g}$ & $a_{g}$ & $b_{1 u}$ & $a_{u}$ \\
$a_{2 g}$ & $b_{1 g}$ & $a_{2 u}$ & $b_{1 u}$ \\
$b_{2 g}$ & $b_{1 g}$ & $b_{2 u}$ & $b_{1 u}$ \\
\hline \hline
\end{tabular}

creases by $34.5 \mathrm{~cm}^{-1}$ upon $S$-T excitation, but in PtP there is no large frequency shift (Tables IV and V). This is connected with the specific character of this mode in FBP; it includes mainly the $\mathrm{C}-\mathrm{N}-\mathrm{H}$ bending vibration.

The out-of-plane $\mathrm{C}-\mathrm{H}$ vibration of the methine bridges could be important in promoting $T_{1}-S_{0}$ intersystem crossing in porphyrins. The out-of-plane $\mathrm{C}_{m}-\mathrm{H}$ vibration in PtP $\left(\nu_{49}, a_{2 u}\right)$ is calculated at $879 \mathrm{~cm}^{-1}$; it has high IR intensity $(I=122 \mathrm{~km} / \mathrm{mole})$ and provides the second intense peak in the IR spectrum of the PtP molecule (Fig. 3; observed at $829 \mathrm{~cm}^{-1}$ ).

In the range of high-frequency $\mathrm{C}-\mathrm{H}$ stretching vibrations there are two close-lying IR bands in the PtP spectrum, at 3215 and $3284 \mathrm{~cm}^{-1}$ (Fig. 3; $e_{u}$ modes $\nu_{95-104}$ in Table IV). This group of IR bands is almost identical to the 3205 and $3279 \mathrm{~cm}^{-1}$ close-lying bands in the IR spectrum of the $\mathrm{ZnP}$ molecule (Table IV).

There are three IR regions (100-600, 900-1000, and $1400-1600 \mathrm{~cm}^{-1}$ ) where vibrational frequencies are metal sensitive. Comparison of IR spectra in the $\mathrm{ZnP}, \mathrm{PdP}$, and PtP series with the FBP vibrational modes ${ }^{27}$ indicates proper frequency shifts for such metal-sensitive IR bands. First of all, the asymmetric $\mathrm{C}_{\alpha} \mathrm{C}_{m}$ stretching vibrations are all sensitive to the size of metal ion. ${ }^{44}$ These are the $\nu_{90}-\nu_{93}$ vibrational modes (Table IV-VII). Their frequencies increase by about $40 \mathrm{~cm}^{-1}$ upon $\mathrm{Zn} \rightarrow \mathrm{Pt}$ substitution. The "gerade" vibrations ( $\left.\nu_{92}, \nu_{93}\right)$ are vibronically active in the $S_{0}-S_{1}$ transition of the $Q$ band (these are $\nu_{19}$ and $\nu_{10}$ in the classification of Ref. 44). In free-base porphin and in $\mathrm{ZnP}$ they are found as the most intense vibronic lines in $Q$-band absorption and fluorescence. ${ }^{28}$ We have obtained similar results for the PtP molecule with the remarkable frequency shift mentioned above. We shall return to the vibronic activity in the next section. 


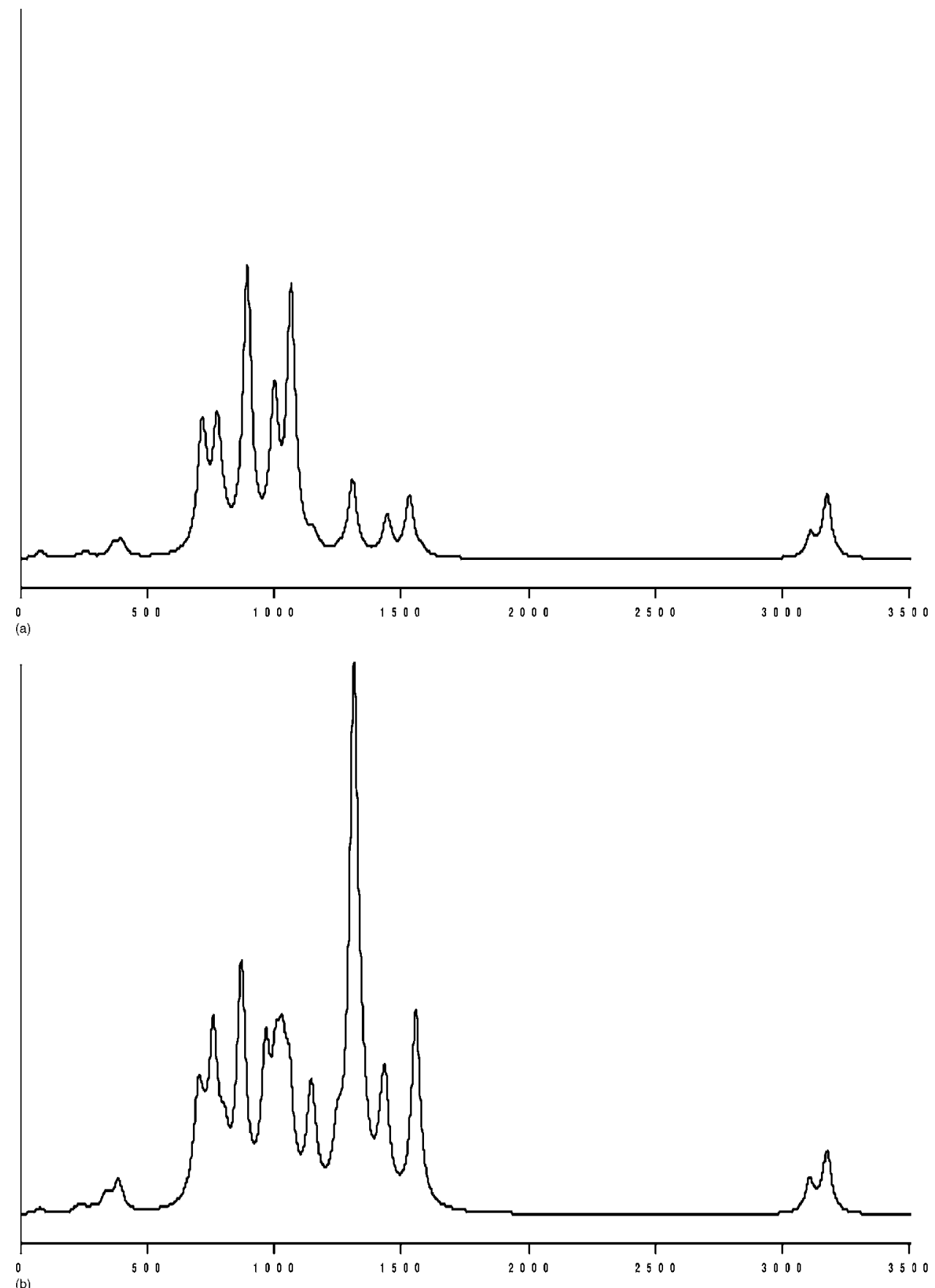

FIG. 3. Infrared absorption spectrum of the PtP molecule calculated by the B3LYP/Lan12DZ method: (a) in the ground state ${ }^{1} A_{g}$ (maximum intensity is $232 \mathrm{~km} / \mathrm{mol}$ ); (b) in the first triplet excited state ${ }^{3} B_{3 u}$ (maximum intensity is $437 \mathrm{~km} / \mathrm{mol}$ ).

The addition of eight alkyl groups at the $\beta$ positions of the pyrrole rings leads to a frequency increase of $\mathrm{C}_{\beta}-\mathrm{C}_{\beta}$ vibrations from $1493 \mathrm{~cm}^{-1}$ in $\operatorname{PtP}\left(\nu_{82,83}\right.$ in Table IV) to $1541 \mathrm{~cm}^{-1}$ in PtOEP. This is the most prominent shift in the IR spectrum upon alkylation. In the Raman spectrum a new line at about $1025 \mathrm{~cm}^{-1}$ is predicted for PtOEP (with a scaling factor of 0.967). It has a large contribution from the ethyl groups. Its Raman activity can be explained by involvement of porphyrin vibrations. Another mixed vibrational mode is shifted from $736 \mathrm{~cm}^{-1}$ in the Raman spectrum of $\operatorname{PtP}\left(\nu_{33}\right.$ in Table VII) to $799 \mathrm{~cm}^{-1}$ upon alkylation. The shift is explained by large involvement of the $\mathrm{C}_{\beta}-\mathrm{C}_{\text {ethyl }}$ bond in this methyl-bridge vibration.

\section{B. Excited states of metalloporphyrins and absorption spectra of PtOEP}

Excitation of free-base porphin, Zn-, and Pd-porphyrins in the Soret band leads to internal conversion to the lowest singlet excited state $S_{1}\left(1^{1} E_{u}\right)$; after that about $5 \%$ of the molecules decay back to the ground state under the emission of fluorescence, while the $S_{1} \rightsquigarrow T_{1}$ intersystem crossing pushes about $90 \%$ of them to the lowest triplet state. ${ }^{17}$ Under the PtP excitation no detectable fluorescence was observed. ${ }^{2,33}$ Thus a proper analysis of phosphorescence should address the singlet excited states. Results of the present calculations of the low-lying excited states and the 


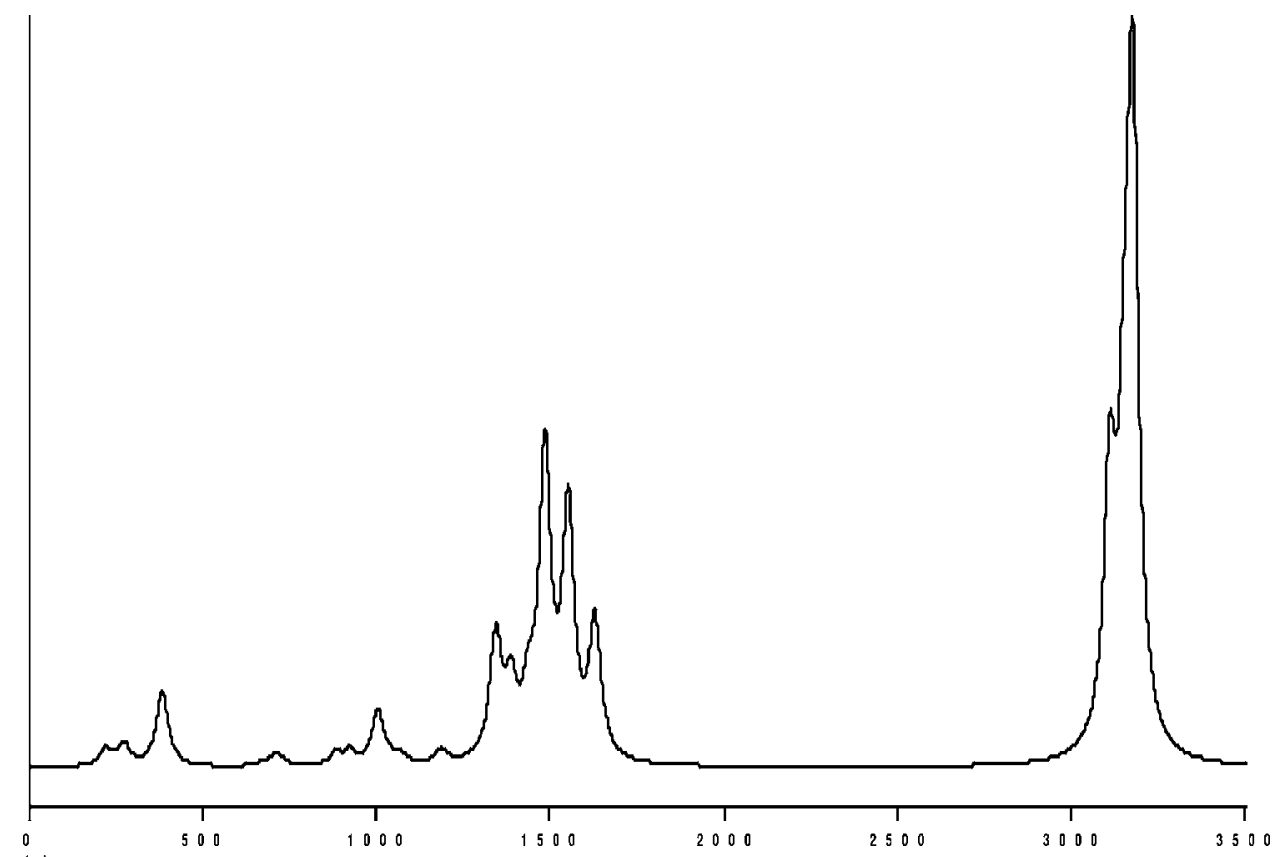

(a)

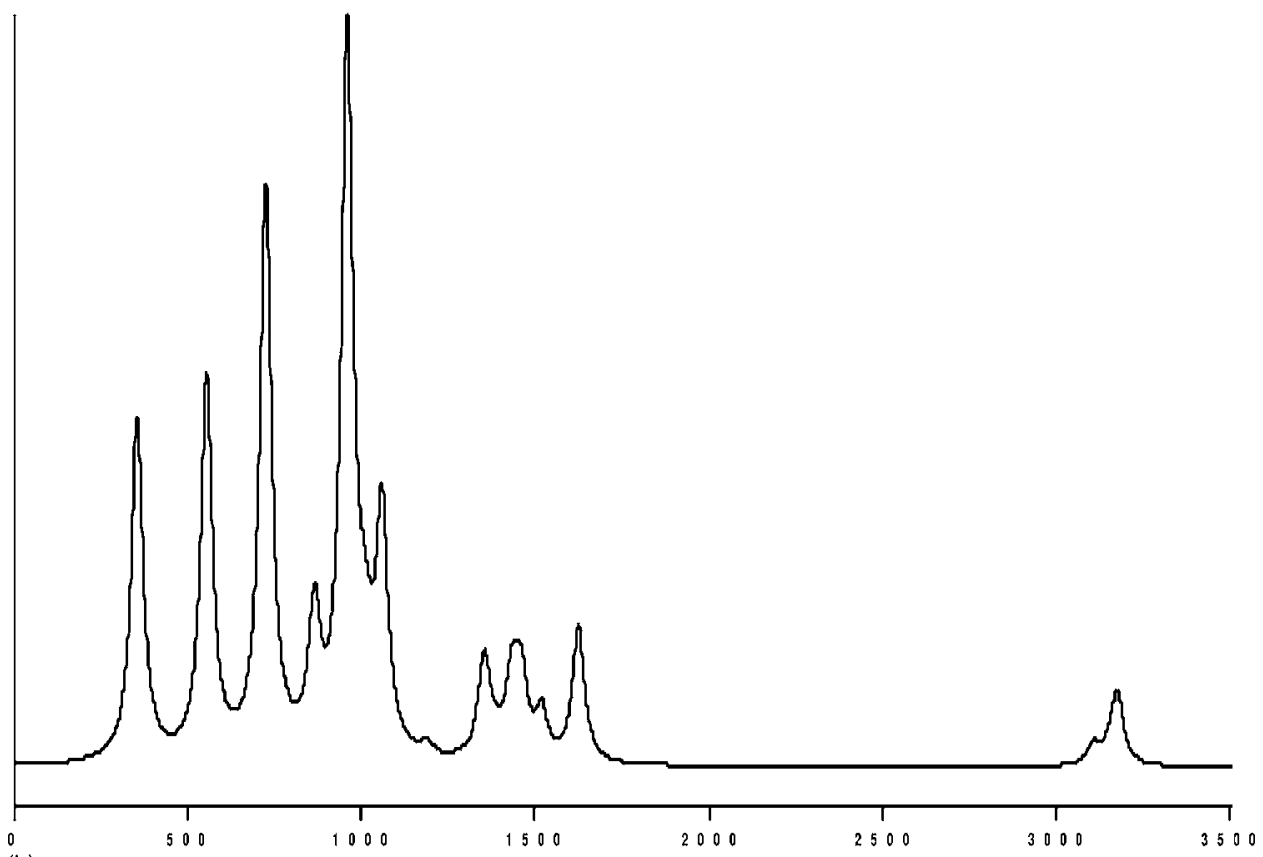

(b)

FIG. 4. Raman spectrum of the PtP molecule calculated by the B3LYP/Lan12DZ method. (a) in the ground state ${ }^{1} A_{g}$ (maximum intensity is $1227 \AA^{4} / a m u$ ); (b) in the first triplet excited state ${ }^{3} B_{3 u}$ (maximum intensity is $9542 \AA^{4} / \mathrm{amu}$ ). amu is atomic mass unit.

$S-S$ absorption spectrum of metal porphyrins are presented in Table I. It is interesting to consider the generic spectral properties of simple metalloporphyrins (ZnP, PdP, and PtP) and compare them with PtOEP spectra. The most important MOs of PtP are given in Fig. 2 and in Table VIII. Finally, the detailed analysis of the excited states of PtP is presented in Tables IX and X.

Gouterman ${ }^{17}$ classified all metalloporphyrins into regular and irregular classes. The regular class contains only closed shell metals while the irregular class contains metals with partly filed $p, d$ shells. For the regular porphyrins the metal has only a small effect on the optical absorption and emission spectra that can be understood as a small perturbation of the $\pi$ electrons of the porphyrin ring. In the case of irregular class the metal orbitals have much stronger effects on absorption and emission of porphyrins, either through much stronger mixing with the porphyrin ring $\pi$ orbitals or through the involvement of new low-lying CT states. ${ }^{17}$ This classification has been based on the old MO theory of Gouterman ${ }^{58}$ and Ake and Gouterman; ${ }^{59}$ it has only been partly checked by modern calculations. ${ }^{49,60-65}$ We do not know of any calculations of optical properties of Pt-porphyrins which refer to an irregular class and are characterized by hypso absorption, which is blueshifted. ${ }^{17}$ All porphyrins have two major absorption bands, namely, the weak $Q$ band in the visible region (500-650 nm) and the $B$ band (or Soret band) in the 
TABLE VII. Raman spectra of Pt-porphyrin and Zn-porphyrin calculated by the B3LYP DFT method with the Lanl2DZ basis set. RSA means Raman scattering activities $\left(\AA^{4} / \mathrm{amu}\right)$, Dep $(\mathrm{P})$-depolarization ratios for plane polarized incident light, Dep(U)-depolarization ratios for unpolarized light, and $\nu_{i}$-frequency $\left(\mathrm{cm}^{-1}\right)$.

\begin{tabular}{|c|c|c|c|c|c|c|c|c|}
\hline \multirow[b]{2}{*}{$i$} & \multirow[b]{2}{*}{ Symmetry } & \multicolumn{4}{|c|}{$\mathrm{PtP}^{\mathrm{a}}$} & \multicolumn{2}{|c|}{$\mathrm{ZnP}^{\mathrm{a}}$} & \multirow{2}{*}{$\frac{\mathrm{ZnP}^{\mathrm{b}}}{\text { Expt. }^{\mathrm{b}}}$} \\
\hline & & $\nu_{i}$ & RSA & $\operatorname{Dep}(\mathrm{P})$ & $\operatorname{Dep}(\mathrm{U})$ & $\nu_{i}$ & RSA & \\
\hline 4,5 & $e_{g}$ & 162.5 & 0.2 & 0.75 & 0.857 & 153.5 & 0.1 & 145.6 \\
\hline 6 & $b_{2 g}$ & 225.9 & 23.7 & 0.75 & 0.857 & 217.6 & 21.5 & \\
\hline 9,10 & $e_{g}$ & 250.7 & 4.7 & 0.75 & 0.857 & 225.9 & 5.1 & 208.5 \\
\hline 11 & $b_{1 g}$ & 284.0 & 34.9 & 0.75 & 0.857 & 198.3 & 27.0 & \\
\hline 18 & $a_{1 g}$ & 397.2 & 123.9 & 0.106 & 0.192 & 364.8 & 100.3 & 363 \\
\hline 21 & $b_{2 g}$ & 428.9 & 1.0 & 0.75 & 0.857 & 420.0 & 0.9 & \\
\hline 22 & $a_{2 g}$ & 443.6 & 0 & 0 & 0 & 413.6 & 0 & 396 \\
\hline 23,24 & $e_{g}$ & 449.7 & 0.3 & 0.75 & 0.857 & 455.6 & 0.3 & \\
\hline 27,28 & $e_{g}$ & 690.2 & 2.2 & 0.75 & 0.857 & 698.2 & 0.9 & \\
\hline 31,32 & $e_{g}$ & 726.1 & 2.0 & 0.75 & 0.857 & 728.0 & 3.0 & \\
\hline 33 & $a_{1 g}$ & 736.8 & 15.5 & 0.15 & 0.264 & 734.5 & 27.5 & 728 \\
\hline 35 & $b_{1 g}$ & 753.2 & 5.7 & 0.75 & 0.857 & 750.7 & 2.4 & \\
\hline 39,40 & $e_{g}$ & 812.0 & 0.9 & 0.75 & 0.857 & 815.2 & 0.6 & \\
\hline 44 & $a_{2 g}$ & 823.0 & 0 & 0 & 0 & 816.8 & 0 & \\
\hline 45 & $b_{2 g}$ & 840.4 & 1.0 & 0.75 & 0.857 & 841.0 & 0.1 & \\
\hline 47,48 & $e_{g}$ & 914.5 & 10.2 & 0.75 & 0.857 & 909.5 & 14.5 & \\
\hline 51,52 & $e_{g}$ & 956.5 & 10.9 & 0.75 & 0.857 & 957.0 & 9.6 & \\
\hline 54 & $a_{2 g}$ & 1026.2 & 0 & 0 & 0 & 1011.0 & 0 & \\
\hline 55 & $b_{1 g}$ & 1030.1 & 1.3 & 0.75 & 0.857 & 1017.9 & 13.5 & \\
\hline 58 & $a_{1 g}$ & 1038.6 & 88.6 & 0.14 & 0.250 & 1010.9 & 137.9 & 995 \\
\hline 61 & $b_{2 g}$ & 1072.4 & 7.8 & 0.75 & 0.857 & 1058.9 & 19.0 & \\
\hline 62 & $b_{1 g}$ & 1099.8 & 4.0 & 0.75 & 0.857 & 1096.8 & 2.6 & \\
\hline 65 & $a_{1 g}$ & 1110.3 & 9.7 & 0.08 & 0.149 & 1101.1 & 9.7 & 1066 \\
\hline 66 & $a_{2 g}$ & 1183.3 & 0.00 & 0.75 & 0.857 & 1179.6 & 0 & \\
\hline 69 & $b_{1 g}$ & 1224.9 & 18.6 & 0.75 & 0.857 & 1196.4 & 53.3 & \\
\hline 70 & $b_{2 g}$ & 1235.3 & 5.5 & 0.75 & 0.857 & 1221.4 & 0.8 & \\
\hline 75 & $a_{2 g}$ & 1370.6 & 0 & 0 & 0 & 1360.6 & 0 & \\
\hline 76 & $b_{2 g}$ & 1389.1 & 196.7 & 0.75 & 0.857 & 1375.8 & 158.0 & 1347 \\
\hline 77 & $a_{2 g}$ & 1390.8 & 0 & 0 & 0 & 1388.2 & 0 & \\
\hline 78 & $a_{1 g}$ & 1394.1 & 2.8 & 0.12 & 0.211 & 1378.6 & 26.5 & 1357 \\
\hline 81 & $b_{1 g}$ & 1434.0 & 111.6 & 0.75 & 0.857 & 1419.0 & 217.5 & $\approx 1385$ \\
\hline 82 & $a_{1 g}$ & 1486.8 & 90.8 & 0.10 & 0.175 & 1465.2 & 135.1 & 1432 \\
\hline 85 & $b_{2 g}$ & 1535.7 & 12.2 & 0.75 & 0.857 & 1505.6 & 14.8 & $\nu_{19}$ \\
\hline 86 & $b_{1 g}$ & 1535.7 & 480.7 & 0.75 & 0.857 & 1526.4 & 374.0 & 1494 \\
\hline 89 & $a_{1 g}$ & 1604.7 & 406.3 & 0.11 & 0.197 & 1583.9 & 367.4 & 1544 \\
\hline 92 & $a_{2 g}$ & 1653.2 & 0 & 0 & 0 & 1615.7 & 0 & \\
\hline 93 & $b_{1 g}$ & 1684.8 & 222.7 & 0.75 & 0.857 & 1666.6 & 205.4 & $\approx 1607$ \\
\hline 94 & $b_{2 g}$ & 3215.0 & 180.6 & 0.75 & 0.857 & 3205.4 & 181.6 & \\
\hline 97 & $a_{1 g}$ & 3215.5 & 244.3 & 0.10 & 0.184 & 3205.6 & 257.1 & \\
\hline 98 & $a_{2 g}$ & 3257.0 & 0 & 0 & 0 & 3253.1 & 0 & \\
\hline 101 & $b_{2 g}$ & 3258.0 & 344.7 & 0.75 & 0.857 & 3253.3 & 379.5 & \\
\hline 102 & $b_{1 g}$ & 3283.8 & 255.8 & 0.75 & 0.857 & 3279.0 & 239.0 & \\
\hline 105 & $a_{1 g}$ & 3284.1 & 894.5 & 0.12 & 0.210 & 3279.4 & 803.9 & \\
\hline
\end{tabular}

near UV region (350-400 nm). In Pt-porphyrins both bands have a hypsochromic shift with respect to $\mathrm{FBP}, \mathrm{MgP}, \mathrm{ZnP}$, and similar (regular) porphyrins. ${ }^{17}$ For PdP, PtP and their derivatives the weak $T_{1} \leftarrow S_{0}$ absorption band is seen at long wavelengths $(610-660 \mathrm{~nm})$. $^{35,64}$

From Table I it is easy to discern the following trend: the $S_{0} \rightarrow T_{1}$ and $S_{0} \rightarrow S_{1}$ transition energies increase with the rise of the metal atomic charge (our calculations reproduce this trend quite well, Tables II). At the same time the CT transition energies (to the $1{ }^{1} A_{2 g}, 1{ }^{1} B_{2 g}$, and $1{ }^{1} E_{g}$ states) decrease along this series. These CT transitions are forbidden in electric dipole approximation and have not been observed, since they are covered by more intense $\pi \rightarrow \pi^{*}$ absorption. But these states and their triplet analogs are important for the $T_{1} \leftarrow S_{0}$ transition intensity calculation.

For the vertical $T_{1} \leftarrow S_{0}$ excitation (the $D_{4 h}$ symmetry of the ground state molecule) the $T_{1}$ excited state has a degenerate ${ }^{1} E_{u}$ character. All spectrally important singlet excited states in this series have ${ }^{1} E_{u}$ symmetry (Tables I) and almost pure $\pi-\pi^{*}$ character of the tertrapyrrole excitation. Thus 


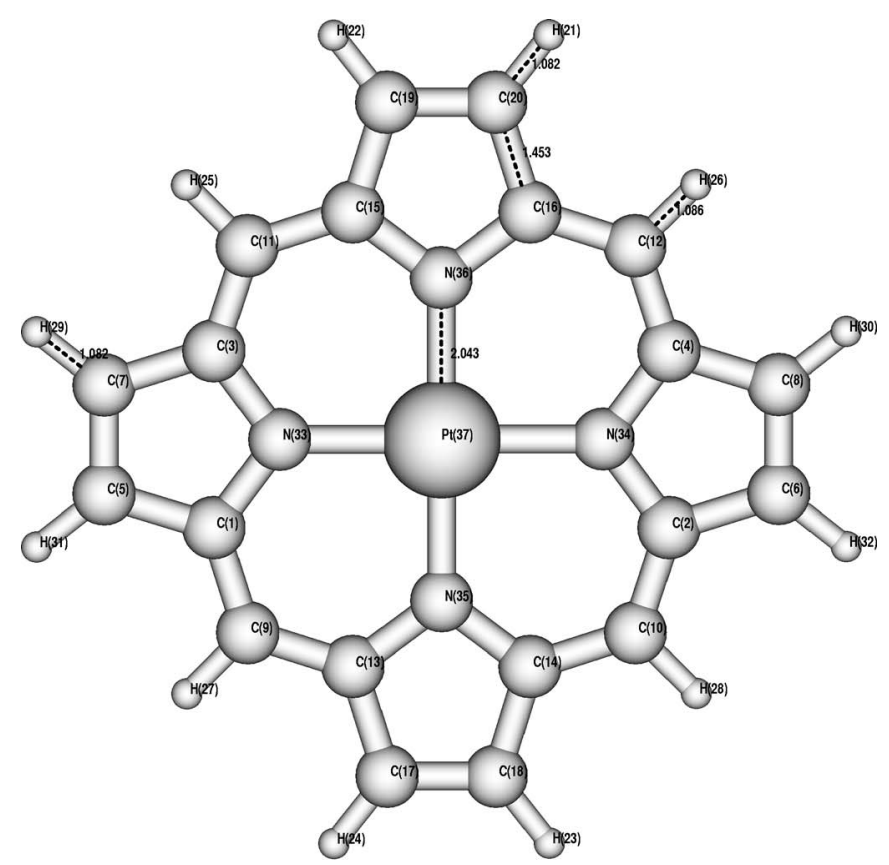

(a)

(b)

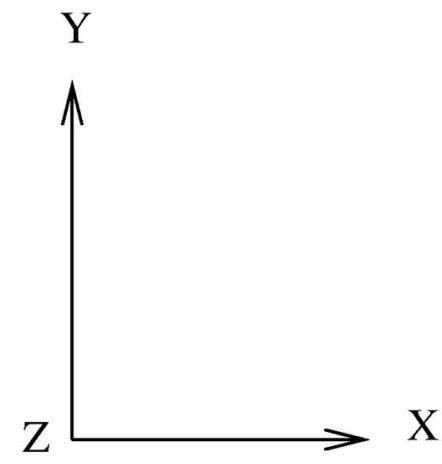

FIG. 5. Numeration of atoms and choice of axes for the platinumporphyrinin molecule.

they can be explained in terms of the Gouterman's fourorbital model $^{58}$ (MO's 88-91 in Table VIII). Only small contributions from other $\pi-\pi^{*}$ excitations are present in the Soret band (Tables X and VIII). A small admixture of the $5 d_{\pi}$ orbitals into the lowest unoccupied molecular orbital (LUMO) of the $5 e_{g}$ type [Table VIII, Fig. 2(e), ] is responsible for the blueshift.

The most important new finding from our calculation is connected with the prediction of the low-lying $1{ }^{1} A_{2 g}$ and $1^{1} B_{2 g}$ states of the $\pi \pi^{*}$ type. They include the $4 e_{g}\left(d_{\pi}\right)$ $\rightarrow 5 e_{g}\left(\pi^{*}\right)$ excitation with considerable charge-transfer character (Tables $\mathrm{X}$ and VIII). The transition to the first such CT state $\left({ }^{1} A_{2 g}\right)$ is only slightly shifted from the $Q$ band in PtP; it is characterized by a very large magnetic dipole transition moment (Table X). The energy of the $1{ }^{1} A_{2 g}$ state goes down with an increase of the metal atomic charge (Table I); in $\mathrm{ZnP}$ it is rather high because the $4 e_{g} \mathrm{MO}$ of the $3 d_{\pi}$ type lies deeper (-0.251 a.u.) among the doubly occupied orbitals than the $4 e_{g}\left(5 d_{\pi}\right) \mathrm{MO}$ of PtP (the inner orbitals of the effective core are not included in the Lanl2DZ basis set. Thus the MO numeration coincides for all metalloporphyrins).
TABLE VIII. Molecular orbitals (MOs) $\left(\psi_{i}\right)$ of the ground state of platinumporphyrin $\left(D_{4 h}\right.$ point group) including correlation with the lower symmetry $\left(D_{2 h}\right.$ point group); MO energy $\left(\epsilon_{i}\right)$ in hartrees.

\begin{tabular}{ccccc}
\hline \hline$i$ & $D_{2 h}$ & $D_{4 h}$ & $\epsilon_{i}$ & Type \\
\hline 80 & $b_{3 g}$ & $3 e_{g}$ & -0.2901 & $\pi$ porphyrin \\
81 & $b_{2 g}$ & $3 e_{g}$ & -0.2901 & $\pi$ porphyrin \\
82 & $b_{1 g}$ & $14 b_{2 g}$ & -0.2830 & $93 \% 5 d_{x y}$ \\
83 & $b_{1 u}$ & $3 a_{2 u}$ & -0.2708 & $\pi$ porphyrin \\
84 & $b_{1 u}$ & $1 b_{2 u}$ & -0.2594 & $\pi$ porphyrin \\
85 & $a_{g}$ & $13 a_{1 g}$ & -0.2366 & $\sigma 5 d_{z}^{2}$ \\
86 & $b_{3 g}$ & $4 e_{g}$ & -0.2178 & $70 \% 5 d_{\pi}$ \\
87 & $b_{2 g}$ & $4 e_{g}$ & -0.2178 & $70 \% 5 d_{\pi}$ \\
88 & $b_{1 u}$ & $4 a_{2 u}$ & -0.2077 & $\pi$ porphyrin \\
89 & $a_{u}$ & $1 a_{1 u}$ & -0.2076 & $\pi$ porphyrin \\
90 & $b_{2 g}$ & $5 e_{g}$ & -0.0891 & $6 \% 5 d_{\pi}+\pi^{*}$ porphyrin \\
91 & $b_{3 g}$ & $5 e_{g}$ & -0.0891 & $6 \% 5 d_{\pi}+\pi^{*}$ porphyrin \\
92 & $a_{u}$ & $2 b_{1 u}$ & -0.0340 & $\pi^{*}$ porphyrin \\
93 & $a_{g}$ & $8 b_{1 g}$ & -0.0007 & $53 \% 5 d_{x^{2}-y^{2}}$ \\
94 & $b_{1 u}$ & $2 b_{2 u}$ & 0.0245 & $\pi^{*}$ porphyrin \\
\hline \hline
\end{tabular}

A similar $S$-S transition has recently been predicted for the $\mathrm{ZnP}$ molecule with $D_{2 h}$ distorted geometry (like in freebase porphin). ${ }^{24}$ In that case the upper state has $1{ }^{1} B_{1 g}$ symmetry and lies even lower than the $Q$ state. The $1{ }^{1} B_{1 g}$ state is really sensitive to distortion along the $b_{1 g}$ mode, which is an active mode for the Jahn-Teller effect. ${ }^{27}$ The $D_{4 h}$ structure of the ground state is distorted into a $D_{2 h}$ rectangle prolongated along the $x$ axis; such distortion occurs not only in the triplet ${ }^{3} E_{u}$ state, but also in the nondegenerate $1{ }^{1} A_{2 g}$ state because of the pseudo-Jahn-Teller effect. ${ }^{24}$

The forbidden $0-0$ transition to the $1{ }^{1} A_{2 g}$ state transforms to an allowed vibronic band under excitation of $e_{u}$ vibrational modes. We have found that the first $e_{u}$ mode $\left(\nu_{7,8}\right.$ in Table IV), which includes the Pt atom displacement from the center, is active in such vibronic mixing. The symmetry of the PtOEP molecule is lower, and selection rules are released. Even a pure electronic transition to the analog of the ${ }^{1} A_{2 g}$ state is now allowed and provides a weak shoulder of the $Q$ band in the absorption spectrum of PtOEP in solid films. 18

Another important state connected with the $4 e_{g}\left(d_{\pi}\right)$ $\rightarrow 5 e_{g}\left(\pi^{*}\right)$ excitation is the third triplet state $\left(1^{3} B_{2 g}\right.$ in Table IX) and also the $1^{3} A_{2 g}$ (Table IX). They are responsible for the strong $T_{1}-T_{n}$ absorption and provide a large SOC integral with the ground singlet state. Thus they give a large contribution to phosphorescence of PtP and PtOEP molecules.

One has to mention the other CT states of the $E_{g}$ symmetry $\left(\sigma \pi^{*}\right)$, which have small $S-T$ splittings (Tables, IX and $\mathrm{X})$. They are connected with the $13 a_{1 g}\left(d_{z^{2}}\right) \rightarrow 5 e_{g}\left(\pi^{*}\right)$ excitation (Table VIII) and are almost pure charge transfer from Pt to the ring. The hypothetical ${ }^{3} E_{g}$ states were thought to be important in explaining the phosphorescence intensityborrowing scheme, proposed by Devolder et al. ${ }^{34}$ but our calculations do not support an exclusive importance of this scheme.

Addition of ethyl groups leads to a redshift in the PtOEP absorption spectrum. The $T_{1}$ state is shifted from $1.94 \mathrm{eV}$ (Table IX) to $1.87 \mathrm{eV}$ in a good agreement with experimental 
TABLE IX. Triplet excited states of platinum-porphyrin, calculated by time-dependent DFT (B3LYP/Lanl2DZ) at the ground state geometry $\left(D_{4 h}\right.$ point group). Correlation with the lower symmetry (the triplet excited state Jahn-Teller distorted structure of the $D_{2 h}$ point group) is also presented. Vertical excitation energy from the $1{ }^{1} A_{1 g}$ ground state $(\Delta E)$ is in $\mathrm{eV}$.

\begin{tabular}{ccccc}
\hline \hline$D_{2 h}$ & $D_{4 h}$ & $\Delta E(\mathrm{eV})$ & $f$ & Determinants \\
\hline $1{ }^{3} B_{1 u}$ & $1{ }^{3} E_{u}$ & 1.943 & $0.000055^{\mathrm{a}}$ & $0.17(88 \rightarrow 90)-0.37(89 \rightarrow 91)-0.1(84 \rightarrow 90)+0.12(80$ \\
& & & & $\rightarrow 92)$ \\
$1{ }^{3} B_{2 u}$ & $1{ }^{3} E_{u}$ & 1.943 & $0.000055^{\mathrm{a}}$ & $\cdots$ \\
$2{ }^{3} B_{2 u}$ & $2{ }^{3} E_{u}$ & 2.252 & $\ldots$ & $\cdots$ \\
$2{ }^{3} B_{3 u}$ & $2{ }^{3} E_{u}$ & 2.252 & $\ldots$ & $0.32(88 \rightarrow 90)+0.66(89 \rightarrow 91)$ \\
$1{ }^{3} B_{1 g}$ & $1{ }^{3} B_{2 g}$ & 2.334 & $\ldots$ & $0.52[(86 \rightarrow 90)+(87 \rightarrow 91)]+0.13(83 \rightarrow 92)-0.12(88$ \\
& & & $0.52[(86 \rightarrow 91)-(87 \rightarrow 90)]$ \\
$1{ }^{3} A_{g}$ & $1{ }^{3} B_{1 g}$ & 2.432 & $\ldots$ & $0.52[(86 \rightarrow 91)+(87 \rightarrow 90)]$ \\
$2{ }^{3} B_{1 g}$ & $1{ }^{3} A_{2 g}$ & 2.454 & $\ldots$ & $0.71(85 \rightarrow 90)$ \\
$2{ }^{3} A_{g}$ & $2{ }^{3} B_{1 g}$ & 2.518 & $\ldots$ & $0.71(85 \rightarrow 91)$ \\
$1{ }^{3} B_{2 g}$ & $1{ }^{3} E_{g}$ & 3.063 & $\mathrm{~b}$ & $-0.50[(86 \rightarrow 90)-(87 \rightarrow 91)]-0.13(84 \rightarrow 92)$ \\
$1{ }^{3} B_{3 g}$ & $1{ }^{3} E_{g}$ & 3.063 & $\mathrm{~b}$ & $\cdots$ \\
$3{ }^{3} B_{2 u}$ & $3{ }^{3} E_{u}$ & 3.342 & $\ldots$ & $0.54(84 \rightarrow 91)-0.34(87 \rightarrow 92)-0.32(83 \rightarrow 91)+0.10(81$ \\
$3{ }^{3} B_{3 u}$ & $3{ }^{3} E_{u}$ & 3.342 & $\ldots$ & $\cdots 92)$ \\
$3{ }^{3} B_{1 g}$ & ${ }^{3} B_{2 g}$ & 3.462 & $\ldots$ & $0.65(88 \rightarrow 92)+0.23[(80 \rightarrow 90)+(81 \rightarrow 91)]$ \\
$3{ }^{3} A_{g}$ & ${ }^{3} B_{1 g}$ & 3.709 & $\ldots$ & $0.68(89 \rightarrow 92)-0.18[(80 \rightarrow 91)+(81 \rightarrow 90)]$ \\
\hline \hline
\end{tabular}

$\overline{{ }^{\mathrm{a}} f \text { is an oscillator strength for the } T_{1} \leftarrow S_{0} \text { absorption, calculated by quadratic response with a combined basis set }}$ as decribed in the text.

${ }^{\mathrm{b}}$ The $T \leftarrow S_{0}$ transition has a charge-transfer character.

data (shift from 2.02 to $1.93 \mathrm{eV}$ ). ${ }^{33}$ The singlet excited states are less shifted. The PtOEP absorption spectrum in the neat thin film has a maximum of the Soret band at $372 \mathrm{~nm}$ and two $Q$ bands at $510 \mathrm{~nm}\left(Q^{\prime}\right)$. A double feature $\left(Q_{1}, Q_{2}\right)$ at $543 \mathrm{~nm}$ is observed for $70-50 \mathrm{~nm}$ thick films. ${ }^{18}$ An increase of the film thickness leads to a reverse of the ratio of the subband maxima $\left(Q_{1} / Q_{2}\right)$ in the $Q$ band but does not change the Soret band. The blueshift and disappearance of the $Q_{1}, Q_{2}$ subband structure are observed in the absorption spectra of PtOEP in toluene solution. ${ }^{18}$ The $Q$ and $Q^{\prime}$ bands reside at 536 and $500 \mathrm{~nm}$, respectively, in solution; we can interpret this splitting $\left(\Delta_{Q} \simeq 1545 \mathrm{~cm}^{-1}\right)$ as the vibronic interval between $0-0$ and $0-1$ bands. The corresponding $b_{1 g}$ vibration in $\operatorname{PtP}\left(\nu_{93}\right.$ in Table VII) is slightly redshifted in the calculated PtOEP Raman spectrum. This asymmetric (out-ofphase) $\mathrm{C}_{\alpha}-\mathrm{C}_{m}$ stretching vibration of methine bridges is very active in vibronic spectra of free-base porphin and $\mathrm{ZnP} ;{ }^{27}$ a similar result is obtained in the present work for PtP

TABLE X. Singlet excited states of platinum-porphyrin, calculated by time-dependent DFT at the ground state geometry with the B3LYP/Lanl2DZ approach $\left(D_{4 h}\right.$ point group). Correlation with the lower symmetry (the triplet excited state Jahn-Teller distorted structure of the $D_{2 h}$ point group) is also presented. The vertical excitation energy from the $1{ }^{1} A_{1 g}$ ground state $(\Delta E)$ is in $\mathrm{eV} ; f$ is an oscillator strength.

\begin{tabular}{ccccc}
\hline \hline$D_{2 h}$ & $D_{4 h}$ & $\Delta E(\mathrm{eV})$ & $f$ & Determinants \\
\hline $1{ }^{1} B_{3 u}$ & $1{ }^{1} E_{u}$ & 2.598 & 0.0033 & $-0.49(88 \rightarrow 90)+0.52(89 \rightarrow 91)$ \\
$1{ }^{1} B_{2 u}$ & $1{ }^{1} E_{u}$ & 2.598 & 0.0033 & $0.49(88 \rightarrow 91)+0.52(89 \rightarrow 90)$ \\
$1{ }^{1} B_{1 g}$ & $1{ }^{1} A_{2 g}$ & 2.668 & $0^{\mathrm{a}}$ & $0.49(86 \rightarrow 90)-0.49(87 \rightarrow 91)$ \\
$2{ }^{1} B_{1 g}$ & $1{ }^{1} B_{2 g}$ & 2.743 & 0 & $0.49(86 \rightarrow 90)+0.49(87 \rightarrow 91)$ \\
$1{ }^{1} A_{g}$ & $1{ }^{1} B_{1 g}$ & 2.790 & 0 & $0.49(89 \rightarrow 90)-0.49(86 \rightarrow 91)$ \\
$1{ }^{1} B_{2 g}$ & $1{ }^{1} E_{g}$ & 3.114 & $0^{\mathrm{b}}$ & $0.70(85 \rightarrow 90)$ \\
$1{ }^{1} B_{3 g}$ & $1{ }^{1} E_{g}$ & 3.114 & $0^{\mathrm{b}}$ & $0.70(85 \rightarrow 91)$ \\
$2{ }^{1} A_{g}$ & $1{ }^{1} A_{1 g}$ & 3.187 & 0 & $0.46[(87 \rightarrow 90)+(86 \rightarrow 91)]$ \\
$2{ }^{1} B_{3 u}$ & $2{ }^{1} E_{u}$ & 3.533 & 0.7157 & $0.39(88 \rightarrow 90)+0.38(89 \rightarrow 91)+0.14(86 \rightarrow 92)+0.13(84$ \\
& & & $\rightarrow 90)$ \\
$2{ }^{1} B_{2 u}$ & $2{ }^{1} E_{u}$ & 3.533 & 0.7158 & $0.39(88 \rightarrow 91)-0.38(89 \rightarrow 90)+0.14(87 \rightarrow 92)-0.13(84$ \\
$3{ }^{1} B_{3 u}$ & $3{ }^{1} E_{u}$ & 4.048 & 0.0675 & $-0.10(88 \rightarrow 90)+0.66(84 \rightarrow 90)$ \\
$3{ }^{1} B_{2 u}$ & $3{ }^{1} E_{u}$ & 4.048 & 0.0677 & $0.10(88 \rightarrow 91)+0.66(84 \rightarrow 91)$ \\
$3{ }^{1} B_{1 g}$ & $2{ }^{1} B_{2 g}$ & 4.282 & 0 & $0.66(88 \rightarrow 92)-0.15[(80 \rightarrow 90)+(81 \rightarrow 91)]$ \\
$3{ }^{1} B_{3 g}$ & $2{ }^{1} E_{g}$ & 4.315 & 0 & $0.63(82 \rightarrow 90)+0.30(86 \rightarrow 93)$ \\
$3{ }^{1} B_{2 g}$ & $2{ }^{1} E_{g}$ & 4.315 & 0 & $0.66(89 \rightarrow 92)-0.30(87 \rightarrow 93)$ \\
$3{ }^{1} A_{g}$ & $2{ }^{1} B_{1 g}$ & 4.324 & 0 & \\
\hline \hline
\end{tabular}

${ }^{\mathrm{a}}$ Magnetic dipole transition moment is very large: $\mu_{z}=1.945 \beta$.

${ }^{\mathrm{b}}$ Magnetic dipole transition moment is equal to $\mu_{x, y}=0.289 \beta$. The state has a big charge-transfer character. 
and PtOEP. In solvents, the Soret band of PtOEP gets narrower and its splitting $\left(\Delta_{B} \simeq 1532 \mathrm{~cm}^{-1}\right)$ becomes apparent. ${ }^{18}$ A similar vibronic explanation could be possible.

A comparison of different methods ${ }^{16,24,27,28}$ indicates that even small basis sets (Lanl2DZ and 3-21G) provide good interpretation of porphyrin absorption spectra in the framework of the TD DFT method with the B3LYP functional. Thus it is reasonable to apply this approximation for our phosphorescence study in the following section.

\section{Phosphorescence}

It is known ${ }^{33}$ that the luminescent properties of porphyrins are far more dependent on metal substitution than are the absorption spectra. The above mentioned CT states are not seen in the absorption since the corresponding transitions from the ground state are electric dipole forbidden. However, the CT states get important for the $T_{1}-S_{0}$ phosphorescence intensity borrowing. The lowest triplet excited state of $\mathrm{ZnP}$, $\mathrm{PdP}$, and PtP is orbitally degenerate $\left({ }^{3} E_{u}\right)$ at the ground state symmetry point group $D_{4 h}$ and should, as such, possess an orbital angular momentum. When studying the phosphorescence, the question arises to which extent the spin is coupled to this orbital angular momentum. ${ }^{17,34,64,65}$ The first analysis of the problem was presented by Gouterman and co-workers. ${ }^{17,33,59,65}$ They pointed out that the ZFS of the six components of the ${ }^{3} E_{u}$ state should represent the importance of SOC relative to the lifting of the orbital degeneracy by the Jahn-Teller effect and by intermolecular interaction with the environment. From magneto-optical experiments on the lower singlet ${ }^{1} E_{u}$ states of $\mathrm{ZnP}$ and $\mathrm{PdP}$, we know that the crystal field lifts the orbital degeneracy and induces a splitting. ${ }^{17}$ Study of the optical detection of magnetic resonance (ODMR) in the $\mathrm{ZnP}$ phosphorescence in an $n$-octan host at $1.2-4.2 \mathrm{~K}$ (Ref. 66) pointed to a single orbital state lacking $D_{4 h}$ symmetry and with three spin components that reflect the usual quantization of the spin in three mutually orthogonal planes. $^{21}$

That means that the Jahn-Teller effect (or the crystal field in previous interpretations ${ }^{64,66}$ ) quenches the orbital angular momentum and splits the ${ }^{3} E_{u}$ state into two components $\left({ }^{3} B_{3 u}\right.$ and ${ }^{3} B_{2 u}$, as follows from our calculations, with the former state being the lowest component; the splitting $\left(\Delta_{E}\right)$ must be so large that at $4.2 \mathrm{~K}$ only the lower ${ }^{3} B_{3 u}$ carries the Boltzmann population. These ideas are consistent with many experiments ${ }^{17,21,67}$ on ODMR studies of phosphorescence of porphyrins without heavy metals and with their theoretical interpretations. ${ }^{23,24,27}$ The ZFS of the phosphorescent triplet states of $\mathrm{MgP}$ and $\mathrm{ZnP}$ observed in ODMR experiments at $4.2 \mathrm{~K}$ proves almost identical to that of the freebase porphin, ${ }^{67}$ in agreement with recent DFT calculations ${ }^{24}$ which show that ZFS is determined by spin-spin coupling and SOC contribution is almost negligible. ${ }^{23,24}$

The EPR study of the $T_{1}$ state of PdP has provided a quite different situation. ${ }^{68}$ The orbital angular momentum is still quenched and $\Delta_{E}=58 \mathrm{~cm}^{-1}$ like in light-metal porphyrins. But with a heavier central metal the SOC contribution to ZFS cannot be ignored; in fact a large SOC integral be- tween two orbital components of the ${ }^{3} E_{u}$ state (denoted as $T_{y}$ and $T_{x}$ ) has been inferred from the EPR analysis ${ }^{68}$

$$
\left\langle T_{y}\left(t^{x}\right)\left|H_{\mathrm{SO}}\right| T_{x}\left(t^{y}\right)\right\rangle=-(i / 2)\left\langle T_{y}\left|\sum_{i, A} \zeta_{A}\left(r_{i, A}\right) l_{i, A}^{z}\right| T_{x}\right\rangle=Z / 2,
$$

where $t^{x}$ is a spin function of spin sublevel with zero projection on the $x$ axis, $l_{i, A}$ denotes the operator for the orbital angular momentum of electron $i$ around nucleus $A$, and the function $\zeta_{A}\left(r_{i, A}\right)$ governs the SOC magnitude at nucleus $A$ and is related to the mean electric field at $r_{i, A}$ distance. For the SOC operator $H_{\mathrm{SO}}=\Sigma_{i, A} \zeta_{A}\left(r_{i, A}\right) \mathbf{l}_{i, A} \cdot \mathbf{s}_{i}$ only one-center integrals are usually accounted for. ${ }^{69}$ In the summation over $A$ the contribution of the heavy $\mathrm{Pd}$ and $\mathrm{Pt}$ atoms dominates. Thus in terms of the four orbital model ${ }^{58}$ the $Z$ integral is reduced to

$$
Z=-i C_{x z}^{2}<5 d_{y z}\left|\zeta_{\mathrm{Pt}}(r) l_{i, \mathrm{Pt}}^{z}\right| 5 d_{x z}>,
$$

where $C_{x z}=C_{y z}$ are coefficients at the $d_{\pi}$ atomic orbitals (AOs) in the expansion of the LUMO $5 e_{g}$. As follows from our DFT calculations this $d_{\pi}$ contribution is not large [Fig. $2(\mathrm{e})]$ which agrees with semiempirical estimations. ${ }^{34,64,68} \mathrm{We}$ have to note that for $\mathrm{ZnP}$ the $3 d_{\pi}$ contribution to the the LUMO $5 e_{g}$ is almost equal to zero; ${ }^{24}$ calculations of the phosphorescence rate with and without $d$ orbitals give similar results. However, besides this expectation value of the SOC operator in the degenerate ${ }^{3} E_{u}$ state [Eq. (15)], there are second order SOC contributions to the ZFS parameter which have not been accounted for in Refs. 34 and 64. As follows from our calculations these get increasingly more important for the PdP and PtP molecules.

For the PtP phosphorescence seven more intriguing level structures were observed in the Zeeman experiment for which no satisfactory explanation could be provided by drawing a simple analogy with the PdP molecule. ${ }^{64}$ In this case the SOC is so strong that the orbital angular momentum $l^{z}$ is only partly quenched: the competition between SOC and the Jahn-Teller effect is then very efficient. The Zeeman experiment on the ${ }^{3} E_{u^{-}}{ }^{1} A_{1 g}$ absorption and emission spectra of PtP in $n$-octane single crystals ${ }^{64}$ clearly demonstrates the importance of the $l^{z}$ quantization. Eigenfunctions of the symmetry operator $P_{R}$ for rotation by $\pi / 2$ about the fourfold axis $z$ for the orbital and spin parts of the total wave function ${ }^{34,64}$ are classified in terms of $K= \pm 1$ and $M_{S}= \pm 1,0$ quantum numbers and also by $J_{z}=K+M_{S}$. If we neglect spin-spin coupling which is really very small in PtP in comparison with the SOC contribution, the six spin sublevels of the ${ }^{3} E_{u}$ state

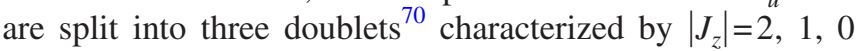
and separated by $\pm Z / 2$. Each doublet corresponds to a particular coupling of the spin and orbital angular momenta; thus in an external magnetic field $\left(H_{z}\right)$ the doublets are split by Zeeman interaction of $H_{z}$ with the resultant magnetic moment of spin $\left(g_{e} \beta\right)$ and orbital $(\Lambda \beta)$ angular momenta. Since $\Lambda \approx 2$ for the lowest triplet state of metalloporphyrins, ${ }^{17}$ the Zeeman interaction is largest for $J_{z}= \pm 2$ when the two moments are parallel, and the Zeeman interaction is very weak for $J_{z}=0$ when the moments quench each other. Such a Zeeman effect is observed in the ${ }^{3} E_{u^{-}}{ }^{1} A_{1 g}$ absorption and emis- 
sion spectra of PtP in $n$-octane single crystal; an additional complication by a weak crystal field which removes the orbital degeneracy of the ${ }^{3} E_{u}$ state should be added. ${ }^{64}$

By these Zeeman effect studies on the phosphorescent state of PdP and PtP molecules in organic host crystals the $Z$ value equal to 15 and $76 \mathrm{~cm}^{-1}$, respectively, were inferred. ${ }^{34,64}$ From our B3LYP calculation with the WTBS basis set for $\mathrm{Pd}$ and $\mathrm{Pt}$ atoms ${ }^{38}$ (3-21G basis set for other atoms) at the optimized $T_{1}$ state geometry (Table III) we have found the $Z$ value equal to 9 and $47 \mathrm{~cm}^{-1}$, respectively. These $Z$ integrals are only approximate, since the ${ }^{3} B_{3 u}$ and ${ }^{3} B_{2 u}$ components are not degenerate at the optimized $T_{1}$ state geometry and the second-order contributions to ZFS are nonnegligible; we have calculated only SOC integrals with the ${ }^{1} A_{u}$ states, which contribute to the final $Z$ value, but the complete analysis of the second-order SOC contribution to ZFS is not possible at the moment.

Now let us turn to the intensity calculation of the $T_{1}-S_{0}$ transition. The parity of $J_{z}$ remains a good quantum number and it may be used for a polarization selection rule of the $T_{1}-S_{0}$ transition: $\Delta J_{z}= \pm 1$ (in-plane polarized) and $\Delta J_{z}= \pm 2$, 0 (out-of-plane polarized). ${ }^{64}$ Using the main idea of Ref. 71 about effective SOC between the lower ${ }^{3} E_{u}$ and ${ }^{1} E_{u}$ states, which is also equal to the $Z$ integral [Eq. (15)], one can predict that in-plane polarization would be strong. This prediction is also confirmed by the Zeeman effect studies on PtP phosphorescence. ${ }^{34,64}$ In spite of a good qualitative agreement of the semiempirical theory predictions ${ }^{59,64,65}$ and the phosphorescence polarization measurements, ${ }^{34}$ our calculations provide some modification of the old scheme, which is essential for understanding the electroluminescence efficiency in organic light emitting diodes.

As first pointed out by Ake and Gouterman ${ }^{59}$ in their study of CuP phosphorescence, the ${ }^{4} E_{u} \rightarrow{ }^{2} B_{1 g}$ emission acquires most of its strength from spin-allowed, in-plane polarized ${ }^{2} E_{u}-{ }^{2} B_{1 g}$ electric dipole transition. (One has to remember that in CuP with its odd number of electrons the ground state is ${ }^{2} B_{1 g}$ doublet.) For PtP the ${ }^{3} E_{u} \rightarrow{ }^{1} A_{1 g}$ phosphorescence should, similarly, be acquired from the ${ }^{1} E_{u}-{ }^{1} A_{1 g}$ transitions, i.e., from the $Q$ and Soret bands. ${ }^{34}$ This process involves first order SOC between ${ }^{1} E_{u}$ and ${ }^{3} E_{u}$ states via the $z$ component of the SOC operator [or $L_{z}$ component of the orbital angular momentum, which provides the $Z$ integral, Eq. (14)]. All these theories ${ }^{34,64,68}$ follow the main idea of Gouterman ${ }^{17}$ that the mixing between the platinum filled $d_{\pi}$ orbital and the porphyrin empty $e_{g}\left(\pi^{*}\right)$ molecular orbital brings strong SOC into the $T_{1}\left(\pi \pi^{*}\right)$ transition (this idea is also mentioned in a recent work ${ }^{72}$ ). Being very fruitful as a qualitative correct guess, this idea suffers in some quantitative details. Namely, the admixture of $d_{\pi}$ orbitals to the $5 e_{g}\left(\pi^{*}\right)$ LUMO (Table VIII and Fig. 5) is not high and does not provide the large SOC mixing between ${ }^{1} E_{u}$ and ${ }^{3} E_{u}$ states via the $z$ component of the SOC operator. Furthermore, the $T_{1}-S_{0}$ intensity borrowing from the $Q$ band is not effective, since the $Q$ band intensity is very low. This contribution to the $T_{1}-S_{0}$ oscillator strength is equal to $7 \times 10^{-5}$ which is much lower than the final value (0.009). At the same time the SOC mixing with the Soret state is almost an order of mag- nitude smaller. Still it provides an essential part of the $T_{1}$ $-S_{0}$ radiative probability $(0.0015)$ in agreement with the idea of Gouterman. ${ }^{17}$

An important additional contribution to the $T_{1}-S_{0}$ transition moment comes from the SOC mixing between the ground state $1{ }^{1} A_{1 g}$ and the triplet $1{ }^{3} A_{2 g}$ state, which is only $2.45 \mathrm{eV}$ above the ground state energy level. The $1{ }^{3} A_{2 g}$ triplet represents mainly the CT excitation $4 e_{g} \rightarrow 5 e_{g}$ (Table IX) from the metal $d_{\pi}$ orbitals $(86,87$ in Table VIII) to the ring $\pi^{*}$ LUMO with some $d_{\pi}$ admixture. The SOC matrix element is calculated to be very high

$$
\left\langle 1^{1} A_{1 g}\left|H_{\text {SO }}^{z}\right| 1^{3} A_{2 g}\left(z^{z}\right)\right\rangle=-i 1786 \mathrm{~cm}^{-1} .
$$

Its absolute value is comparable to the SOC integral between the lowest ${ }^{3} D_{2}$ and ${ }^{1} D_{2}$ states of the neutral Pt atom $\left(3945 \mathrm{~cm}^{-1}\right)$ which agree well with a mean-field calculation in Ref. 41 and with our previous multireferences configuration interaction ECP study. ${ }^{73}$ The ground state of the Pt atom ${ }^{3} D_{3}\left[5 d^{9}\left({ }^{2} D_{2.5}\right) 6 s\right]$ is quite close to the ${ }^{3} D_{2}$ state of the same configuration (the gap is equal to $776 \mathrm{~cm}^{-1}$ ) ${ }^{74}$ because of strong SOC-induced repulsion of the ${ }^{3} D_{2}$ and ${ }^{1} D_{2}$ states. ${ }^{73}$ The upper spin sublevel ${ }^{74}$ of the same triplet, ${ }^{3} D_{1}$, is as high as $10132 \mathrm{~cm}^{-1}$, which illustrates the large importance of SOC and the deviation from the Lande interval rule in $\mathrm{Pt}$ atom. ${ }^{73}$ The present SOC calculation agrees with the atomic multiplet structure studied before, ${ }^{73}$ thus we cannot consider the result of Eq. (16) as an artifact.

Coming back to PtP we have to stress that the triplettriplet $1{ }^{3} A_{2 g} \leftarrow 1{ }^{3} E_{u}$ transition is characterized by a very big electric dipole transition moment $\left(0.92 e a_{0}\right)$. The presence of the $1{ }^{3} A_{2 g}$ state admixture in the ground singlet state leads to the phosphorescence intensity borrowing from the triplettriplet $1{ }^{3} A_{2 g}-1{ }^{3} E_{u}$ transition with in-plane polarization This channel is the main one for the $t^{z}$ spin sublevel. Following the ideas of Refs. 24, 54, 64, and 65, one has to consider the SOC mixing between ${ }^{1} E_{u}$ and $1{ }^{3} E_{u}$ states as the main mechanism of the phosphorescence intensity borrowing from the $Q$ and Soret bands. But this spin-orbit coupling is not so effective as the above mentioned SOC with the CT state and $T$-T intensity borrowing: $1{ }^{3} E_{u} \leftarrow 1{ }^{3} A_{2 g} \leftarrow H_{\mathrm{SO}}^{Z} \rightarrow 1{ }^{1} A_{1 g}$.

Involvement of the $\pi \pi^{*}$ CT state $1^{3} A_{2 g}$ in the phosphorescence theory of the PtP dye is very essential for a consistent explanation of electroluminescence in the light emitting diodes and the triplet exciton involvement after electrical injection in the conductive band. The low energy of the CT state $1{ }^{3} A_{2 g}$ in platinum porphyrin (Table I) explains not only the high radiative efficiency of the dye phosphorescence, but also a strong interaction between the triplet state of the guest dye and the triplet excitons in the conductive band of the organic host polymer.

In Table II the $T_{1} \rightarrow S_{0}$ transition moments and radiative rate constants (Einstein coefficient for spontaneous emission) calculated by the quadratic response TD DFT/B3LYP method with different basis sets are presented (most results are obtained with the $3-21 \mathrm{G}$ basis). All basis sets predict ${ }^{23,75}$ extremely weak phosphorescence of free-base porphin (Table II) with unusually low rate constants for spontaneous emission. This is in general agreement with all available experi- 
mental data. ${ }^{22,76,77}$ Only one spatial component of the ${ }^{3} E_{u}$ degenerate state is presented in Table II for metal porphyrins.

The same results are obtained for the other spatial component; in this case the $T^{z}$ spin sublevel should emit light with the in-plane $y$ polarization. A large change of spinsublevel selectivity and phosphorescence polarization is calculated for Pt-porphyrin in comparison with FBP and $\mathrm{MgP}$ molecules. The more active spin sublevel is $T^{z}$ in PtP which provides the predominant in-plane polarization of the $T_{1}$ $\rightarrow S_{0}$ transition. This is in agreement with photoselection and Zeeman experiments on the PtP phosphorescence in organic crystals. $^{34}$

\section{Vibronic bands in phosphorescence}

A prominent doublet at about $230 \mathrm{~cm}^{-1}$ appears in the ${ }^{3} E_{u} \rightarrow{ }^{1} A_{1 g}$ phosphorescence spectra of $\mathrm{PdP}$ and $\mathrm{PtP}$; it is also seen in the ${ }^{4} E_{u} \rightarrow{ }^{2} B_{1 g}$ emission of CuP. PtOEP shows a relatively strong peak at about $280 \mathrm{~cm}^{-1}$ to the red of the main $0-0$ band in nonane at liquid nitrogen. ${ }^{33}$ The peak can be attributed to a vibrational mode of $e_{g}$ symmetry on the ground of its in-plane polarization in PtP crystals. ${ }^{34,64}$ From our calculations we attribute it to $\nu_{9,10}$ modes in PtP (Table VII; the calculated frequency is $250.8 \mathrm{~cm}^{-1}$ ). In PtOMP and PtOEP molecules, the calculated frequency of this mode is shifted to $269 \mathrm{~cm}^{-1}$. In PtOMP these are the $\nu_{29,30}$ modes, which are quite similar to PtP and $\mathrm{ZnP}$ vibrations (Table VII). These are out-of-plane and out-of-phase vibrations of nitrogen atoms with corresponding deformation of methine bridges. They also include out-of-plane and out-of-phase bending vibrations of the $\mathrm{CH}_{3}$ groups. One has to note that the out-of-plane $e_{g}$ vibrations are not very intense in Raman spectra of metalloporphyrins and are not well studied. ${ }^{44,47}$ They borrow their Raman activity through vibronic coupling of $E_{u}$ and $A_{2 u}$ states, which have in-plane and out-of-plane polarizations for transitions from the ground state. The offdiagonal components of a polarizability tensor derivative $\left(\alpha_{x z}=\alpha_{y z}\right)$ are responsible for the Raman intensity of the $e_{g}$ vibrations. Since the Raman intensity is weak, one cannot expect strong vibronic coupling of the ${ }^{1} E_{u}$ and ${ }^{1} A_{2 u}$ states. This scheme was proposed in Ref. 34 as one of the main roots of intensity borrowing in PtP phosphorescence. Now this scheme can be eliminated.

In PtOMP there are many $\mathrm{CH}_{3}$ vibrations in the lowfrequency region (hindered rotation and deformations) which are between the first two $e_{g}$ modes predicted in the PtP spectrum (Table VII). The first $e_{g}$ mode $\left(\nu_{4,5}\right.$ in Table VII) is strongly shifted to $82 \mathrm{~cm}^{-1}$ in PtOMP and involves the outof-plane deformations of the $\mathrm{CH}_{3}$ groups. It is not active in PtOMP phosphorescence in agreement with our calculation.

In PtP phosphorescence of the doped $n$-octane single crystal there are three intense vibronic lines at 238, 640, and $694 \mathrm{~cm}^{-1}$. ${ }^{34}$ We have attributed the latter two to the $e_{g}$ modes $\nu_{27,28}$ and $\nu_{31,32}$, respectively (Table VII); we have to mention that the Lanl2DZ basis set overestimates the vibrational frequency in this region by about $20-30 \mathrm{~cm}^{-1}$. The calculations predict very low Raman activity of the latter two modes
(Table VII); thus they have not been observed even in a well-studied Raman spectra of ZnP. All these modes correspond to out-of-plane vibrations.

In the triplet state they are slightly split into $b_{2 g}$ and $b_{3 g}$ frequencies, but they are not changed in the nature. At the same time the QR calculations indicate very high vibronic activity of these modes in the ${ }^{3} E_{u} \rightarrow{ }^{1} A_{1 g}$ phosphorescence spectra of both PdP and PtP molecules. The $e_{g}$ vibronic lines emitted from the $t^{z}$ spin sublevel are mostly out-of-plane polarized in agreement with experiment. ${ }^{34}$ The $238 \mathrm{~cm}^{-1}$ mode provides the most intense vibronic feature in PtP phosphorescence in the doped crystal. ${ }^{34}$ It is also the most active in our QR calculation, since it includes the out-of-plane Pt-N vibrations which strongly influence the $5 d \mathrm{AO}$ mixing and SOC integrals.

Devolder et al $^{34}$ have proposed two hypotheses for the perturbation scheme of $e_{g}$ vibronic activity in PtP phosphorescence. The first one includes SOC between $1{ }^{3} E_{u}$ and the singlet intermediate state ${ }^{1} I_{u}$ with intensity borrowing from transitions in the singlet system

$$
{ }^{3} E_{u} \leftarrow H_{\mathrm{SO}}^{x, y} \rightarrow{ }^{1} I_{u} \leftarrow H_{V} \rightarrow\left({ }^{1} E_{u}, e_{g}\right) \rightarrow\left({ }^{1} A_{1 g}, e g\right)
$$

Here $H_{V}$ denotes vibronic perturbation and $\left({ }^{1} E_{u}, e_{g}\right)$ stands for a vibronic state in which the $e_{g}$ vibration is excited with one quantum. ${ }^{34}$ The second hypothesis is based on SOC between $1{ }^{1} A_{1 g}$ and the triplet intermediate state ${ }^{3} E_{u}$ with intensity borrowing from transitions in the triplet manifold

$$
{ }^{3} E_{u} \rightarrow{ }^{3} I_{g} \leftarrow H_{V} \rightarrow\left({ }^{3} E_{g}, e_{g}\right) \leftarrow H_{\mathrm{SO}}^{x, y} \rightarrow\left({ }^{1} A_{1 g}, e_{g}\right) .
$$

Our calculations indicate that the first hypothesis should be eliminated; there are no low-energy states of ${ }^{1} A_{1 u},{ }^{1} A_{2 u}$, ${ }^{1} B_{1 u}$, and ${ }^{1} B_{2 u}$ symmetries (Table $\mathrm{X}$ ) which could simulate the hypothetic ${ }^{1} I_{u}$ state in the scheme of Eq. (17). The SOC integral with the lowest ${ }^{1} A_{1 u}$ state is negligible. From the analysis of relative decay rates of the two lower spin sublevels of the ${ }^{3} E_{u}$ state, in an external magnetic field, to the two components of $e_{g}$ vibration Devolder et $a .^{34}$ have concluded that the second scheme is more appropriate. The present DFT calculations support this conclusion, but there are differences in details, which specify the hypothesis, presented by Eq. (18). In Fig. 6 of Ref. 34 Devolder et al. have considered the ${ }^{3} A_{1 g}$ state produced by the $1 a_{1 u} \rightarrow 2 a_{1 u}$ excitation as a candidate for the hypothetic ${ }^{3} I_{g}$ state in the scheme of Eq. (18). This choice does not correspond to our calculations. The empty $2 a_{1 u}$ orbital is rather high in energy (0.062 a.u.) and the ${ }^{3} A_{1 g}$ state is not close to the $1^{3} E_{g}$ level, as it was proposed in Ref. 34 (it is above $6.5 \mathrm{eV}$ ). Instead of this proposal we have to consider the $1{ }^{3} A_{2 g}$ state in order to simulate the hypothetic ${ }^{3} I_{g}$ state in the scheme of Eq. (18). The importance of the $1^{3} A_{2 g}$ state of CT type was already discussed above (Eq. (16)). We need to add one quantum of the $e_{g}$ vibration in the ${ }^{3} E_{u}$ state and then the $T$-T transition $1{ }^{3} A_{2 g}$ $-\left({ }^{3} E_{u}, e_{g}\right)$ becomes $z$ polarized. The scheme Eq. (18) should be modified in the manner:

$$
\left({ }^{3} E_{u}, e_{g}\right) \rightarrow\left({ }^{3} E_{g}, e_{g}\right) \leftarrow H_{V} \rightarrow{ }^{3} I_{g} \leftarrow H_{\mathrm{SO}}^{x, y} \rightarrow\left({ }^{1} A_{1 g}, e_{g}\right) .
$$


Thus vibronic lines of the $e_{g}$ type become polarized in another direction in comparison with the $0-0$ phosphorescent emission which agree with observations. ${ }^{34}$

\section{CONCLUSIONS}

We have presented time-dependent DFT and quadratic response calculations of the phosphorescence spectra of platinum(II) octaethyl porphyrin (PtOEP) and platinum(II) porphyrin (PtP) which elucidate the role of charge-transfer states, spin-orbit coupling (SOC), and Jahn-Teller effect in the triplet-singlet $(T-S)$ transition intensity of PtOEP. This molecule has been used as a guest dye in organic light emitting diodes (LED) in order to convert triplet excitons (usually spent to heating in LED devices based on conjugated organic polymers) into light emission. ${ }^{2}$ The heavy metal porphyrins offer a possibility to enhance the electroluminescence efficiency of LED and to overcome the limit imposed by formation of triplet excitons due to (i) relatively strong SOC in such a heavy metal atom such as Pt and (ii) because of a special role played by a charge transfer state of $d_{\pi}$ $\rightarrow \pi^{*}$ type in the $T_{1} \rightarrow S_{0}$ intensity borrowing scheme and in the exciton hopping.

The mechanism of SOC-induced phosphorescence of metalloporphyrins proposed earlier ${ }^{17,59}$ is here only partly confirmed. In an aromatic molecule, phosphorescence has a long lifetime and is out-of-plane polarized, since SOC between $T$ and $S \pi \pi^{*}$ states in hydrocarbons is negligible. ${ }^{14}$ This implies spin restriction on electroluminescence in organic polymers because electron-hole recombination produces $75 \%$ triplet and $25 \%$ singlet excited states, the former being dark.

In metalloporphyrins SOC between $1{ }^{3} E_{u}$ and ${ }^{1} E_{u}$ states of $\pi \pi^{*}$ type was proposed to be relatively large because of $d_{\pi^{-}}$AO admixture into the LUMO $e_{g}\left(\pi^{*}\right)$ (59) which provides phosphorescence intensity borrowing from the $Q$ and Soret bands. Our calculations indicate that this SOC is relatively weak especially for the Soret state. More important is the SOC-induced admixture of the ${ }^{3} A_{2 g}$ charge-transfer state of $d_{\pi} \rightarrow \pi^{*}$ type into the ground state and the phosphorescence intensity borrowing from $T-T$ transition ${ }^{3} A_{2 g}-1{ }^{3} E_{u}$.

The IR and Raman spectra in the $S_{0}$ and $T_{1}$ states are here also studied for proper assignment of vibronic bands in phosphorescence. An orbital angular momentum of the $T_{1}$ state is not quenched completely by the Jahn-Teller effect. A large zero-field splitting (ZFS) is obtained for PtP and PtOEP which results from competition between SOC and JahnTeller effects in agreement with results from studies of EPR and Zeeman spectra. ${ }^{34,64}$ A strong vibronic activity of PtP phosphorescence is found for the $e_{g}$ mode at $230 \mathrm{~cm}^{-1}$. In the PtOEP molecule the calculated frequency of this mode is shifted to $270 \mathrm{~cm}^{-1}$ in agreement with phosphorescence measurements. ${ }^{33}$ These out-of-plane and out-of-phase vibrations of nitrogen atoms with corresponding deformation of methine bridges involve some bending of the ethyl groups, which explains the frequency shift in PtOEP. Such vibrations include also out-of-plane distortion of Pt-N bonds and create a strong SOC perturbation. Thus they produce considerable change in radiative constants of different spin sublevels of the triplet state; they also promote the $S_{1} \rightarrow T_{1}$ intersystem crossing.

The TD DFT calculations explain the most important features of phosphorescence of porphins with closed- $d$-shell $\left(3 d^{10} 4 s^{2}, \mathrm{ZnP}\right)$ and open- $d$-shell $\left(5 d^{9} 6 s^{1}, \mathrm{PtP}\right)$ transition metal atoms. Spin-sublevel selectivity of the singlet-triplet transitions allows us to predict a magnetic field effect in electroluminescence of dye-doped LED devices at low temperature.

\section{ACKNOWLEDGMENTS}

This work has been supported by the Swedish Research Council and the Wenner-Gren Foundation.

${ }^{1}$ M. A. Baldo, D. O'Brien, M. A. Thompson, and S. R. Forrest, Phys. Rev. B 60, 14422 (1999).

${ }^{2}$ M. A. Baldo and S. R. Forrest, Phys. Rev. B 62, 10958 (2000a).

${ }^{3}$ Z. Shuai, D. Beljonne, R. Silbey, and J. L. Bredas, Phys. Rev. Lett. 84, $131(2000)$.

${ }^{4}$ J. S. Wilson, A. S. Dhoot, A. J. Seeley, M. S. Khan, A. Kohler, and R. H. Friend, Nature (London) 413, 828 (2001).

${ }^{5}$ M. Wohlgenannt, K. Tandon, S. Mazumdar, S. Ramasesha, and Z. V. Vardeny, Nature (London) 409, 494 (2001).

${ }^{6}$ Y. Y. Noh, C. L. Lee, J. J. Kim, and K. Yase, J. Chem. Phys. 118, 2853 (2003).

${ }^{7}$ Y. A. Serebrennikov and B. F. Minaev, Chem. Phys. 114, 359 (1987).

${ }^{8}$ U. Steiner and T. Ulrich, Chem. Rev. (Washington, D.C.) 89, 51 (1989).

${ }^{9}$ Y. A. Serebrennikov, R. R. Mukhin, B. F. Minaev, and Z. M. Muldahmetov, Teor. Eksp. Khim. 22, 203 (1986).

${ }^{10}$ Y. A. Serebrennikov, B. F. Minaev, and B. A. Abdrahmanov, Khim. Fiz. 6, 799 (1987).

${ }^{11}$ Y. A. Serebrennikov, B. F. Minaev, and R. R. Mukhin, Zh. Fiz. Khim. 63, 730 (1989).

${ }^{12}$ Z. M. Muldahmetov, B. F. Minaev, and G. A. Ketsle, Optical and Magnetic Properties of the Triplet State (Nauka, Alma Ata, 1983).

${ }^{13}$ D. Beljonne, Z. Shuai, G. Pourtois, and J. L. Bredas, J. Phys. Chem. A 105, 3899 (2001).

${ }^{14}$ S. P. McGlynn, T. Azumi, and M. Kinoshita, Molecular Spectroscopy of the Triplet State (Engelwood Cliffs, NJ, 1969).

${ }^{15}$ J. B. Birks, Photophysics of Aromatic Molecules (Wiley-Interscience, London, 1970).

${ }^{16}$ B. F. Minaev, Spectrochim. Acta. Part A 60, 3213 (2004).

${ }^{17}$ M. Gouterman, in The Porphyrins, edited by D. Dolphin (Academic, New York, 1978), Vol. III, p. 1.

${ }^{18}$ J. Kalinowski, W. Stampor, and J. Szmytkowski, J. Chem. Phys. 122, 154710 (2005).

${ }^{19}$ M. A. Baldo and S. R. Forrest, Phys. Rev. B 62, 10967 (2000b).

${ }^{20}$ B. F. Minaev, Izv. Vyssh. Uchebn. Zaved. Fiz. 9, 12 (1978).

${ }^{21} \mathrm{R}$. Connors and W. Leenstra, in Triplet State ODMR Spectroscopy (Wiley, New York, 1982), p. 258.

${ }^{22}$ M. P. Tsvirko, K. N. Solovjev, A. T. Gradyushko, and S. S. Dvornikov, Opt. Spectrosc. 38, 400 (1975).

${ }^{23}$ O. Loboda, I. Tunnell, B. Minaev, and H. Ågren, Chem. Phys. 312, 299 (2005).

${ }^{24}$ B. F. Minaev and A. B. Minaev, Opt. Spectrosc. 98, 248 (2005).

${ }^{25}$ H. Ågren, O. Vahtras, and B. Minaev, Adv. Quantum Chem. 27, 71 (1996).

${ }^{26}$ I. Tunnell, Z. Rinkevicius, P. Salek, O. Vahtras, B. T. Helgaker, and H. Ågren, J. Chem. Phys. 119, 11024 (2003).

${ }^{27}$ B. Minaev and H. Ågren, Chem. Phys. 315, 215 (2005).

${ }^{28}$ B. F. Minaev, Y.-H. Wang, C.-K. Wang, Y. Luo, and H. Ågren, Spectrochim. Acta, Part A 65, 308 (2006).

${ }^{29}$ W. Kohn and L. J. Sham, Phys. Rev. Lett. 52, 997 (1984).

${ }^{30}$ W. Kohn and L. J. Sham, Phys. Rev. 140, A1133 (1965).

${ }^{31}$ R. Bauernschmitt and R. Ahlrichs, Chem. Phys. Lett. 256, 454 (1996).

${ }^{32}$ J. Olsen and P. Jørgensen, J. Chem. Phys. 82, 3235 (1985).

${ }^{33}$ D. Eastwood and M. Gouterman, J. Mol. Spectrosc. 35, 359 (1970).

${ }^{34}$ P. Devolder, N. V. Dijk, and J. H. van der Waals, Mol. Phys. 43, 335 (1981). 
${ }^{35}$ A. Albrecht, J. Chem. Phys. 38, 354 (1963).

${ }^{36}$ P. J. Hay and W. R. Wadt, J. Chem. Phys. 82, 299 (1985).

${ }^{37}$ R. Krishnan, J. S. Binkley, R. Seeger, and J. A. Pople, J. Chem. Phys. 72 650 (1980).

${ }^{38}$ S. Huzinaga and M. Klobukowski, Chem. Phys. Lett. 212, 260 (1993).

${ }^{39}$ P. Macak, Y. Luo, and H. Ågren, Chem. Phys. Lett. 330, 447 (2000).

${ }^{40}$ S. Koseki, M. H. Schmidt, and M. S. Gordon, J. Phys. Chem. A 102, 10430 (1998)

${ }^{41}$ C. Marian and U. Wahlgren, Chem. Phys. Lett. 251, 357 (1996).

${ }^{42}$ L. K. Stoll, M. Z. Zgierski, and P. M. Kozlowski, J. Phys. Chem. A 106, 170 (2002).

${ }^{43}$ D. P. Piet, D. Danovich, H. Zuilhof, and E. J. R. Sudholter, J. Chem. Soc., Perkin Trans. 2 1999, 1653.

${ }^{44}$ X.-Y. Li and M. Z. Zgierski, J. Phys. Chem. 95, 4268 (1991).

${ }^{45}$ E. J. Baerends, G. Ricciardi, A. Rosa, and S. J. A. van Gisbergen, Coord Chem. Rev. 230, 5 (2002).

${ }^{46}$ N. Verdal, P. M. Kozlowski, and B. S. Hudson, J. Phys. Chem. A 109, 5724 (2005).

${ }^{47}$ A. A. Jarzecki, P. M. Kozlowski, P. Pulay, B.-H. Ye, and X.-Y. Li, Spectrochim. Acta, Part A 53, 1195 (1997).

${ }^{48}$ K. A. Nguyen, P. N. Day, and R. Pachter, J. Phys. Chem. A 104, 4748 (2000).

${ }^{49}$ K. A. Nguyen, P. N. Day, and R. Pachter, J. Chem. Phys. 110, 9135 (1999a).

${ }^{50}$ D. Lide, in CRC Handbook in Chemistry and Physics (CRC, Boca Raton, FL, 1993)

${ }^{51}$ J. Hoard, in Porphyrins and Metaloporphyrins, edited by K. M. Smith (Elsevier, New York, 1975), p. 317.

${ }^{52}$ L. L. Gladkov and K. N. Solovyov, Spectrochim. Acta, Part A 41, 1437 (1985).

${ }^{53}$ L. L. Gladkov and K. N. Solovyov, Spectrochim. Acta, Part A 42, 1 (1986).

${ }^{54}$ P. M. Kozlowski, A. A. Jarzecki, and P. Pulay, J. Phys. Chem. 100, 7007 (1996a).

${ }^{55}$ P. M. Kozlowski, A. A. Jarzecki, P. Pulay, X.-Y. Li, and M. Z. Zgierski, J. Phys. Chem. 100, 13985 (1996b).
${ }^{56}$ T. Schröder, R. Schinke, M. Ehara, and K. Yamashita, J. Chem. Phys. 109, 6641 (1998)

${ }^{57}$ B. Heumann and R. Schinke, J. Chem. Phys. 101, 7488 (1994).

${ }^{58}$ M. Gouterman, J. Mol. Spectrosc. 6, 138 (1961).

${ }^{59}$ R. L. Ake and M. Gouterman, Theor. Chim. Acta 15, 20 (1969).

${ }^{60}$ K. A. Nguyen, P. N. Day, and R. Pachter, J. Phys. Chem. A 103, 9378 (1999b).

${ }^{61}$ K. A. Nguyen and R. Pachter, J. Chem. Phys. 114, 10757 (2001).

${ }^{62}$ M. Liao and S. Scheiner, J. Chem. Phys. 117, 205 (2002).

${ }^{63}$ P. M. Kozlowski, T. G. Spiro, A. Berces, and M. Z. Zgierski, J. Phys. Chem. B 102, 2603 (1998).

${ }^{64}$ N. V. Dijk, M. Noort, S. Volker, G. W. Canters, and J. H. van der Waals, Chem. Phys. Lett. 71, 415 (1980).

${ }^{65}$ M. Gouterman, B. S. Yamanashi, and A. L. Kwiram, J. Chem. Phys. 56, 4073 (1972)

${ }^{66}$ I. Y. Chan, W. G. van Dorp, T. J. Schaafsma, and J. H. van der Waals, Mol. Phys. 22, 741 (1971)

${ }^{67}$ J. A. Kooter and J. H. van der Waals, Mol. Phys. 37, 997 (1979).

${ }^{68}$ J. A. Kooter and J. H. van der Waals, Mol. Phys. 33, 1545 (1977).

${ }^{69}$ B. F. Minaev, Izv. Vyssh. Uchebn. Zaved. Fiz. 5, 93 (1971).

${ }^{70}$ M. Perrin, M. Gouterman, and C. Perrin, J. Chem. Phys. 56, 2492 (1972).

${ }^{71}$ M. Perrin, M. Gouterman, and C. Perrin, J. Chem. Phys. 50, 4137 (1969).

${ }^{72}$ G. E. Khalil, A. Chang, M. Gouterman, J. B. Callis, L. R. Dalton, N. J. Turro, and S. Jockusch, Rev. Sci. Instrum. 76, 054101 (2005).

${ }^{73}$ B. F. Minaev and H. Ågren, J. Mol. Catal. A: Chem. 149, 179 (1999).

${ }^{74}$ C. Moore, Atomic Energy Levels (NBS, Washington, DC, 1958), Vol. III.

${ }^{75}$ B. F. Minaev, D. Jonsson, P. Norman, and H. Ågren, Chem. Phys. 194, 19 (1995).

${ }^{76}$ M. Gouterman and G. E. Khalil, J. Mol. Spectrosc. 53, 88 (1974).

${ }^{77}$ W. G. van Dorp, W. H. Schoemaker, M. Soma, and J. H. van der Waals, Mol. Phys. 30, 1701 (1975).

${ }^{78}$ L. Edwards, D. H. Dolphin, M. Gouterman, and A. D. Adler, J. Mol. Spectrosc. 38, 16 (1971).

${ }^{79}$ K. N. Solovyev, L. L. Gladkov, A. S. Staruhin, and S. F. Shkirman, Spectroscopy of Porphyrins: virational states (Nauka i Tehnika, Minsk, 1985) (in Russian). 